\title{
Multiscale elastic-viscoplastic computational analysis
}

\section{A detailed example}

\author{
Nicolas Relun - David Néron — Pierre-Alain Boucard \\ LMT-Cachan (ENS Cachan/CNRS/UPMC/PRES UniverSud Paris) \\ 61 avenue du Président Wilson, F-94235 Cachan cedex \\ relun,neron,boucard@Imt.ens-cachan.fr
}

ABSTRACT. The objective of this work is to develop an efficient strategy for quasi-static problems with elastic-viscoplastic constitutive laws. Our approach is based on the multiscale LATIN method for domain decomposition, and particularly on the use of the Proper Generalized Decomposition (PGD) method, which allows a drastic decrease in computation costs. We present the method in its general form applicable to problems with constitutive laws expressed using internal variables; then we discuss the technical features which are necessary in order to deal with elastic-viscoplastic models. We illustrate the method in detail through a onedimensional example using a Chaboche-type elastic-viscoplastic constitutive law.

RÉSUMÉ. L'objectif de ce travail est de développer une stratégie efficace pour le traitement de problèmes élasto-viscoplastiques d'évolution. Notre approche est fondée sur la méthode multiéchelle de décomposition de domaine LATIN, et plus particulièrement sur la méthode Proper Generalized Decomposition (PGD) qui permet de réduire notablement les coûts de calcul. La méthode est présentée de manière générale pour les problèmes avec une loi de comportement matériau à variables internes; ensuite les détails techniques induits par l'introduction d'une loi élasto-viscoplastique sont discutés. La méthode est expliquée en détails sur un exemple unidimensionnel avec une loi matériau élasto-viscoplastique de type Chaboche.

KEYWORDS: LATIN, PGD, elastic-viscoplastic, DDM.

MOTS-CLÉS : LATIN, PGD, élasto-viscoplastique, DDM.

DOI:10.3166/EJCM.20.379-409 (c) 2011 Lavoisier, Paris 


\section{Introduction}

The types of problems considered in this paper originate from a project named APPROFI, supported by the French National Research Agency and associating SNECMA (SAFRAN Group) and several other research laboratories and SMEs. The objective of this project is to derive a new methodology in order to predict the behavior of aerospace engine components whose material behavior at high temperature is described appropriately by an elastic-viscoplastic model. The simulation of such problems leads to time-dependent nonlinear analyses which can be computationally expensive when using standard methods, such as a direct approach based on a NewtonRaphson algorithm.

In order to reduce this computation cost, the most popular method today consists in using parallel computing associated with a domain decomposition technique (see, for instance, (Gosselet et al., 2006; Magoulès et al., 2006) for a review). One group of methods used to deal with quasi-static nonlinear problems is known as the "Newton-Krylov-Schur" (NKS) family. These methods consist in using Newton-type algorithms to deal with the nonlinearity aspects, thus leading to sequences of linear systems condensed at the interfaces, which makes parallel computing possible (a review of these techniques can be found in (Wriggers, 2008)). Some of these methods are very popular, such as the Balanced Domain Decomposition (BDD) method (Le Tallec et al., 1991; Mandel, 1993) or the Finite Element Tearing and Interconnecting (FETI) method (Farhat et al., 1991; Farhat et al., 2000a). Multiscale techniques have been introduced to increase the convergence rate of these iterative methods, as in (Gosselet et al., 2002) with the extension of classical preconditioners to the BDD framework, or (Farhat et al., 1995) with the introduction of coarse models into the FETI method to deal with time-dependent problems. These strategies enable the efficient resolution of many physical problems (thanks to the robustness of Newton-type methods) with large numbers of degrees of freedom (thanks to the efficiency of domain decomposition methods) (Farhat et al., 2000b; Risler et al., 2000). To increase computational efficiency, numerous techniques are based on the resolution of a homogenized problem related to macro quantities which can be interpreted as the average part of the micro ones. The key issue is the transfer of information from one scale to the other. A very efficient strategy for linear periodic media consists in applying the homogenization theory introduced by Sanchez-Palencia (Sanchez-Palencia, 1974), for which additional developments and related computational approaches can be found in (Feyel, 2003; Devries et al., 1989; Zohdi et al., 1996; Oden et al., 1999; Fish et al., 1997).

Another approach to dealing with nonlinearities relies on the nonincremental LATIN strategy (Ladevèze, 1999), which has already been used for nonlinear material behavior (Boisse et al., 1990; Boisse et al., 1991; Ladevèze, 1992; Cognard et al., 1993; Cognard et al., 1999) and for large strains (Aubard et al., 2002; Boucard et al., 1997). The LATIN strategy enables the introduction of a two-scale domain decomposition method allowing parallel processing (Ladevèze et al., 2002b). A key feature of the LATIN strategy is that at each iteration an approximate solution is avail- 
able over the entire space-time domain. In particular, the solution can be sought at each iteration using separated variables (i.e. a finite sum of products of temporal functions by spatial functions), a technique introduced initially in (Ladevèze, 1985) and now called Proper Generalized Decomposition (Néron et al., 2010; Chinesta et al., 2008; Nouy, 2007; Ammar et al., 2006) which leads to a drastic reduction in computation time (Nouy et al., 2004; Ladevèze et al., 2010). The PGD technique has already been used in (Cognard et al., 1999; Ladevèze, 1992; Ladeveze et al., 2002a) in the case of viscoplastic materials. Since that study, a more robust version restricted to viscoelastic materials was presented in (Ladevèze et al., 2010). Here, we extend this robust version to the case of viscoplastic materials.

This extension leads to the introduction of new difficulties:

- The search directions are chosen to ensure an optimal convergence of the method. This leads to choose tangent directions linked to the viscoplastic behaviour law (as it is done in classical Newtion algortihm). The operators associated to the tangent direction are defined in this paper.

- These optimal directions induce time and iteration dependent operators. It was not the case for the viscoelastic material. This has heavy consequences on the numerical implementation of the method, as numerous simplifications are no more possible.

- Furthermore solving the coarse problem is more tricky as it depends on the search directions.

That why we choose to explain in detail the numerical implementation of the method using a one-dimensional example in the case of viscoplastic materials.

The paper is organized as follows. Section 2 introduces the reference problem, with particular emphasis on the formulation of the constitutive law in order to ensure that the state laws are linear. A general presentation of the multiscale method is given in Section 3 and its application to a one-dimensional elastic-viscoplastic analysis is detailed in Section 4. A more complex 3D academic example is presented in the last section.

\section{Description of the problem}

\subsection{The reference problem}

For the sake of simplicity, let us consider the quasi-static isothermal evolution of a structure defined over the space-time domain $[0, T] \times \Omega$, assuming small perturbations. (For much more general types of material behavior, see (Ladevèze, 1999).) The structure is subjected to prescribed body forces $\underline{f}_{d}$, to traction forces $\underline{F}_{d}$ over a part $\partial_{2} \Omega$ of the boundary, and to prescribed displacements $\underline{U}_{d}$ over the complementary part $\partial_{1} \Omega$ (see Figure 1 ).

The state of the structure is defined by the set of fields $\mathbf{s}=\left(\dot{\varepsilon}_{\mathrm{p}}, \dot{\mathbf{X}}, \boldsymbol{\sigma}, \mathbf{Y}\right)$ (where $\square$ denotes the derivative with respect to time), in which: 


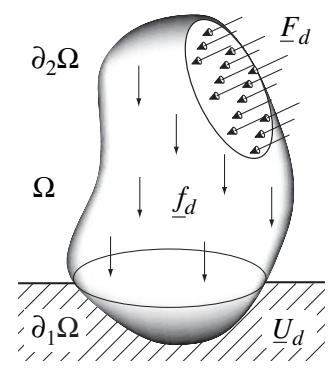

Figure 1. The reference problem in domain $\Omega$

$-\dot{\varepsilon}_{\mathrm{p}}$ designates the inelastic part of the strain field $\varepsilon$ corresponding to the displacement field $\underline{U}$, uncoupled into an elastic part $\varepsilon_{\mathrm{e}}$ and an inelastic part $\varepsilon_{\mathrm{p}}=\varepsilon-\varepsilon_{\mathrm{e}} ; \mathbf{X}$ designates the remaining internal variables;

$-\sigma$ designates the Cauchy stress field and $\mathbf{Y}$ the set of variables conjugate of $\mathbf{X}$.

All these quantities are defined over the space-time domain $[0, T] \times \Omega$ and assumed to be sufficiently regular. Introducing the following notations for the primal and dual fields:

$$
\mathbf{e}_{\mathrm{p}}=\left[\begin{array}{c}
\varepsilon_{\mathrm{p}} \\
-\mathbf{X}
\end{array}\right], \mathbf{e}_{\mathrm{e}}=\left[\begin{array}{c}
\varepsilon_{\mathrm{e}} \\
\mathbf{X}
\end{array}\right], \mathbf{e}=\left[\begin{array}{c}
\varepsilon \\
0
\end{array}\right], \mathbf{f}=\left[\begin{array}{c}
\sigma \\
\mathbf{Y}
\end{array}\right]
$$

the mechanical dissipation rate is:

$$
\operatorname{Tr}\left[\boldsymbol{\sigma} \dot{\varepsilon}_{\mathrm{p}}\right]-\mathbf{Y} \cdot \dot{\mathbf{X}}=\dot{\mathbf{e}}_{\mathrm{p}} \circ \mathbf{f}
$$

where - denotes the contraction associated with the tensorial nature of $\mathbf{X}$ and $\mathbf{Y}$, and $\circ$ denotes the corresponding operator.

Following (Ladevèze, 1999), a "normal" formulation is used for the constitutive law. In this context, normality, which is required for the method described later, has the following definition:

\section{Definition}

The formulation of a constitutive model is said to be normal if it can be written as:

$$
\begin{aligned}
\mathbf{f} & =\mathbf{A e}_{\mathrm{e}} \\
\dot{\mathbf{e}}_{\mathrm{p}} & =\mathbf{B}(\mathbf{f}), \quad \mathbf{e}_{\mathrm{p}}=0 \quad \text { at } t=0
\end{aligned}
$$

where $\mathbf{A}$ is a positive symmetric tensor (linear operator) which is constant with respect to time, and $\mathbf{B}$ is a material-dependent, possibly nonlinear operator which must be positive in order to verify the second law of thermodynamics.

$$
\dot{\mathbf{e}}_{\mathrm{p}} \circ \mathbf{f} \geqslant 0
$$


This type of formulation is very general and, in the absence of damage, can be written for most materials.

\subsection{Formulation of the constitutive model}

Let us take as an example the Marquis-Chaboche elastic-viscoplastic constitutive model (Lemaitre et al., 1994), which is commonly used for metallic materials at high temperatures. In this model,

- the primal variable $\mathbf{e}_{\mathrm{p}}$ consists of the plastic strain $\varepsilon_{\mathrm{p}}$, and the location $\alpha$ of the center of the elastic domain, i.e. $\mathbf{X}=\boldsymbol{\alpha}$,

- the dual variable $\mathrm{f}$ consists of the stress $\sigma$, and the back stress $\boldsymbol{X}$ associated with $\alpha$.

The constitutive model is defined by a free energy of the form:

$$
\rho \Psi\left(\varepsilon_{\mathrm{e}}, \boldsymbol{\alpha}, p\right)=\frac{1}{2} \operatorname{Tr}\left[\mathbf{C} \varepsilon_{\mathrm{e}} \varepsilon_{\mathrm{e}}\right]+\frac{1}{3} \operatorname{Tr}[\mathcal{C} \boldsymbol{\alpha} \boldsymbol{\alpha}]
$$

where $\mathbf{C}$ is the Hooke's tensor (with $E$ the Young modulus, $\nu$ the Poisson's ratio), and $\mathcal{C}$ a constant (positive or zero) tensor. Then, the state equations are:

$$
\begin{gathered}
\boldsymbol{\sigma}=\rho \frac{\partial \Psi}{\partial \boldsymbol{\varepsilon}_{\mathrm{e}}}=\mathbf{C} \boldsymbol{\varepsilon}_{\mathrm{e}} \\
\boldsymbol{X}=\rho \frac{\partial \Psi}{\partial \boldsymbol{\alpha}}=\frac{2}{3} \mathcal{C} \boldsymbol{\alpha}
\end{gathered}
$$

With the previous notations, the state laws can be written as:

$$
\mathbf{f}=\mathbf{A e}_{\mathrm{e}}
$$

where $\mathbf{A}$ is a linear operator. The elastic domain $f$ is defined as:

$$
f=(\boldsymbol{\sigma}-\boldsymbol{X})_{e q}-\sigma_{y}
$$

where $\sigma_{y}$ is the yield stress and $(\boldsymbol{\sigma}-\boldsymbol{X})_{e q}=J_{2}(\boldsymbol{\sigma}-\boldsymbol{X})$ is Von Mises' equivalent stress. The definition of a non-associative potential $F$ leads to the flow direction:

$$
F=(\boldsymbol{\sigma}-\boldsymbol{X})_{e q}+\frac{3 \gamma}{4} \operatorname{Tr}\left[\mathcal{C}^{-1} \boldsymbol{X} \boldsymbol{X}\right]
$$

where $\gamma$ is a material property. Viscosity is taken into account through the Norton law with a power function, leading to the plastic multiplier $\Phi$ :

$$
\Phi(f)=k\langle f\rangle_{+}^{\mathcal{N}}, \text { where } k=\frac{1}{K^{\mathcal{N}}}
$$

$n$ and $K$ being material parameters. Then, the evolution equations are:

$$
\frac{d}{d t}\left[\begin{array}{c}
\varepsilon_{\mathrm{p}} \\
-\boldsymbol{\alpha}
\end{array}\right]=k\langle f\rangle_{+}^{\mathcal{N}}\left[\begin{array}{c}
\frac{3}{2}\left(\boldsymbol{\sigma}^{D}-\boldsymbol{X}\right) /(\boldsymbol{\sigma}-\boldsymbol{X})_{e q} \\
-\frac{3}{2}\left(\boldsymbol{\sigma}^{D^{2}}-\boldsymbol{X}\right) /(\boldsymbol{\sigma}-\boldsymbol{X})_{e q}+\frac{3 \gamma}{2} \mathcal{C}^{-1} \boldsymbol{X}
\end{array}\right]
$$


where $\sigma^{D}$ denotes the deviatoric part of the stress. This expression is of the form

$$
\dot{\mathrm{e}}_{\mathrm{p}}=\mathbf{B}(\mathbf{f})
$$

In this usual formulation of the Marquis-Chaboche model, $\mathbf{A}$ is linear and, therefore, the formulation is normal. If an isotropic hardening is added, the state law becomes non-linear in the usual formulation of the Marquis-Chaboche model. A change of variable described in (Ladevèze, 1999) enables to get back to a linear state law and then to a normal formulation.

\section{The multiscale computational strategy}

This section gives only a brief presentation of the multiscale computational strategy. For more details, see (Nouy et al., 2004; Ladevèze et al., 2010). The interested reader can refer to (Ladevèze et al., 2007) for a description of the method in the discretized framework and a comparison with other decomposition techniques.

\subsection{Substructuring of the problem}

The structure is viewed as an assembly of simple components, namely substructures and interfaces (Ladevèze, 1999) (see Figure 2). Concerning time, the domain $I=[0, T]$ is coarsely divided into a small number of subintervals $I_{i}^{C}=\left[t_{i}^{C}, t_{i+1}^{C}\right]$.
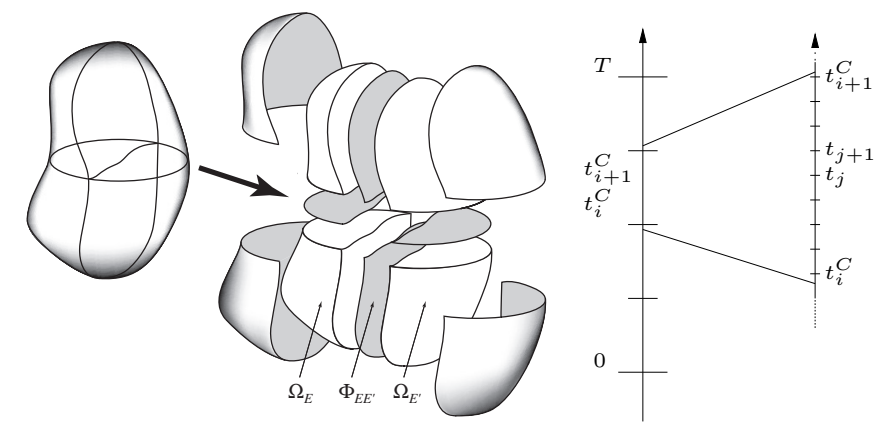

Figure 2. Decomposition of $\Omega$ into substructures $\Omega_{E}$ and interfaces $\Phi_{E E^{\prime}}$, and of $I=[0, T]$ into coarse subintervals $I_{i}^{C}=\left[t_{i}^{C}, t_{i+1}^{C}\right]$

Let $\Phi_{E E^{\prime}}$ denote the interface between a substructure $\Omega_{E} \prod^{1}$ and a substructure $\Omega_{E^{\prime}}$ (Figure 2). Each substructure and each interface has its own variables and its own equations (admissibility, equilibrium and constitutive relation) which control its evolution. The state of a substructure $\Omega_{E}$ is defined entirely by $\dot{\mathbf{e}}_{\mathrm{p} E}$ and $\mathbf{f}_{E}$, the restrictions

1. The notation $\square_{E}$ is used to indicate the restriction of a quantity $\square$ to substructure $\Omega_{E}$. 
of fields $\dot{\mathbf{e}}_{\mathrm{p}}$ and $\mathbf{f}$ to $\Omega_{E}$. The state of an interface $\Phi_{E E^{\prime}}$ is defined by $\left(\underline{W}_{E}, \underline{W}_{E^{\prime}}\right)$, the restriction of the displacement field to $\Phi_{E E^{\prime}}$, and $\left(\underline{F}_{E}, \underline{F}_{E^{\prime}}\right)$, the restriction of the normal stress to $\Phi_{E E^{\prime}}$.

A relation among these quantities is introduced in order to characterize the behavior $\mathbf{b}_{E E^{\prime}}$ of the interface:

$$
\mathbf{b}_{E E^{\prime}}\left(\underline{\dot{W}}_{E}, \underline{\dot{W}}_{E^{\prime}}, \underline{F}_{E}, \underline{F}_{E^{\prime}}\right)=0
$$

For example, for a perfect interface, $\underline{\dot{W}}_{E}=\underline{\dot{W}}_{E^{\prime}}$ and $\underline{F}_{E}+\underline{F}_{E^{\prime}}=\underline{0}$. Other types of complex interface behavior which can be easily taken into account using this mixed decomposition method can be found in (Ladevèze et al., 2001). This is a capital property of the strategy which enables various types of behavior to be described easily.

Let $\mathbf{s}_{E}=\left(\dot{\mathbf{e}}_{\mathrm{p} E}, \dot{W}_{E}, \mathbf{f}_{E}, \underline{F}_{E}\right)$ denote the set of the fields describing the state of substructure $\Omega_{E}$ and its boundary $\partial \Omega_{E}\left(\mathbf{E}_{E}, \mathcal{W}_{E}, \mathbf{F}_{E}\right.$ and $\mathcal{F}_{E}$ denoting the corresponding spaces). For the sake of simplicity, we will limit the presentation to the case of zero initial conditions, except for the initial displacement $\underline{U}_{E \mid t=0}$. Taking into account other types of initial conditions is not difficult.

\subsection{Admissibility conditions for substructure $\Omega_{E}$}

Let us introduce the following spaces and the corresponding vector spaces (denoted $\left.\square^{\star}\right)$ :

- the space $\mathcal{U}_{E}$ of the kinematically admissible fields $\left(\underline{U}_{E}, \underline{\underline{W}}_{E}\right)$ such that

$$
\underline{U}_{E \mid \partial \Omega_{E}}=\underline{W}_{E} \quad \underline{U}_{E \mid t=0}=\underline{U}_{E 0}
$$

- the space $\mathcal{F}_{E}$ of the statically admissible fields $\left(\mathbf{f}_{E}, \underline{F}_{E}\right)$ such that $\forall\left(\underline{U}_{E}^{\star}, \underline{W}_{E}^{\star}\right) \in \mathcal{U}_{E}^{\star}$

$$
\begin{aligned}
-\int_{I_{i}^{C} \times \Omega_{E}} \boldsymbol{\sigma}_{E} & : \varepsilon\left(\underline{\dot{U}}_{E}^{\star}\right) d \Omega d t \\
& +\int_{I_{i}^{C} \times \Omega_{E}} \underline{f}_{d} \cdot \underline{\dot{U}}_{E}^{\star} d \Omega d t+\int_{I_{i}^{C} \times \partial \Omega_{E}} \underline{F}_{E} \cdot \underline{\underline{W}}_{E}^{\star} d S d t=0
\end{aligned}
$$

- the space $\mathcal{E}_{E}$ of the kinematically admissible fields $\left(\dot{\mathbf{e}}_{E}, \dot{W}_{E}\right)$ such that

$$
\begin{aligned}
\exists\left(\underline{U}_{E}, \underline{W}_{E}\right) & \in \mathcal{U}_{E}, \quad \dot{\varepsilon}_{E}=\nabla_{s y m} \underline{\dot{U}}_{E}, \quad \forall\left(\mathbf{f}_{E}^{\star}, \underline{F}_{E}^{\star}\right) \in \mathcal{F}_{E}^{\star}, \\
& -\int_{I_{i}^{C} \times \Omega_{E}} \boldsymbol{\sigma}_{E}^{\star}: \dot{\varepsilon}_{E} d \Omega d t+\int_{I_{i}^{C} \times \partial \Omega_{E}} \underline{F}_{E}^{\star} \cdot \underline{\dot{W}}_{E} d S d t=0
\end{aligned}
$$

- the space $\mathbf{A}_{\mathbf{d} E}$ of the " $E$-admissible" variables $\mathbf{s}_{E}$ such that $\left(\mathbf{A}_{E}^{-1} \dot{\mathbf{f}}_{E}+\right.$ $\left.\dot{\mathbf{e}}_{\mathrm{p} E}, \underline{W}_{E}\right) \in \mathcal{E}_{E}$ and $\left(\mathbf{f}_{E}, \underline{F}_{E}\right) \in \mathcal{F}_{E}$ 


\subsection{A two-scale description of the unknowns}

Our multiscale approach consists in introducing a two-scale description of the unknowns involving a "macro" scale $\left(\square^{M}\right)$ and a "micro" scale $\left(\square^{m}\right)$. Macroquantities are mean values over space and time. For $\underline{\dot{W}}_{E} \in \mathcal{W}_{E}$, the macro part $\underline{\dot{W}}_{E}^{M}$ and its micro complement $\underline{\dot{W}}_{E}^{m}$ are defined by $\forall \underline{F}^{M \star} \in \mathcal{F}_{E}^{M}$ :

$$
\int_{I_{i}^{C} \times \partial \Omega_{E}}\left(\underline{\dot{W}}_{E}^{M}-\underline{\dot{W}}_{E}\right) \cdot \underline{F}^{M \star} d S d t=0 \quad \text { and } \quad \underline{\dot{W}}_{E}^{m}=\underline{\dot{W}}_{E}-\underline{\dot{W}}_{E}^{M}
$$

Spaces $\mathcal{F}_{E}^{M}$ and $\mathcal{W}_{E}^{M}$ can be chosen arbitrarily. In practice, these spaces are defined by the linear parts of the forces and displacements in space and the corresponding quadratic parts in time. An important point of the strategy, which gives it its multiscale character, is the choice of the admissibility conditions for the macroquantities. The set of the macroforces $\underline{F}^{M}=\left(\underline{F}_{E}^{M}\right)_{\Omega_{E} \subset \Omega}$ must a priori verify the transmission conditions systematically, including the boundary conditions. The corresponding subspace of $\mathcal{F}^{M}=\otimes \mathcal{F}_{E}^{M}$ is denoted $\mathcal{F}_{a d}^{M}$. The subspace of $\mathcal{F}$ whose elements have their macro parts in $\mathcal{F}_{a d}^{M}$ is denoted $\mathcal{F}_{a d}$. We use the same definition for $\mathcal{W}^{M}, \mathcal{W}_{a d}^{M}$ and $\mathcal{W}_{a d}$.

\subsection{The LATIN method}

The cornerstone of the strategy is the LATIN method (Ladevèze, 1999). First, one introduces two spaces: the space $\mathbf{A}_{\mathbf{d}}$ of the elements which verify the global linear equations: $\mathbf{s}=\left(\mathbf{s}_{E}\right)_{\Omega_{E} \subset \Omega} \in \mathbf{A}_{\mathbf{d}}$ if $\mathbf{s}$ verifies

a) the $E$-admissibility condition: $\forall \Omega_{E} \subset \Omega, \quad \mathbf{s}_{E} \in \mathbf{A}_{\mathbf{d} E}$

b) the admissibility of the macroforces: $\underline{F} \in \mathcal{F}_{\text {ad }}$

and the space $\boldsymbol{\Gamma}$ of the elements which verify the local nonlinear equations: $\hat{\mathbf{s}}=$ $\left(\hat{\mathbf{s}}_{E}\right)_{\Omega_{E} \subset \Omega} \in \boldsymbol{\Gamma}$ if $\hat{\mathbf{s}}$ verifies

c) the evolution law in $\Omega_{E}: \forall \Omega_{E} \subset \Omega, \quad \hat{\mathbf{e}}_{\mathrm{p} E}=\mathbf{B}\left(\hat{\mathbf{f}}_{E}\right), \quad \hat{\mathbf{e}}_{\mathrm{p} E}(t=0)=0$

d) the interface behavior: $\forall \Phi_{E E^{\prime}} \mathbf{b}_{E E^{\prime}}\left(\underline{\hat{\dot{W}}}_{E}, \underline{\hat{\dot{W}}}_{E^{\prime}}, \underline{\hat{F}}_{E}, \underline{\hat{E}}_{E^{\prime}}\right)=0$

The state law and the initial conditions are verified by the solutions of both $\mathbf{A}_{\mathbf{d}}$ (thanks to the normal formulation of the constitutive law) and $\boldsymbol{\Gamma}$. Clearly, the exact solution of the decomposed problem is defined by sexact $\in \mathbf{A}_{\mathbf{d}} \cap \boldsymbol{\Gamma}$. Taking advantage of the interesting properties of the equations which constitute $\mathbf{A}_{\mathbf{d}}$ and $\boldsymbol{\Gamma}$ (i.e. globality in space, but linearity in the former case; nonlinearity, but locality defined at each integration point in the latter case), the problem is solved using a two-stage iterative scheme. Each iteration of the solver consists of:

- a first stage, called the "linear stage", in which linear problems which are global in the space variable are solved; 
- a second stage, called the "local stage", in which nonlinear problems are solved at each integration point.

The linear and local stages are named in reference to the linearity (respectively the locality) of the problems to be solved. Each of these stages results in an element of $\boldsymbol{\Gamma}$ and $\mathbf{A}_{\mathbf{d}}$ alternatively. In order to close the problem, one needs to introduce what we call the "search directions" $\mathbf{E}^{+}$and $\mathbf{E}^{-}$, whose choice will be studied later. Finally, one can take advantage of the space-time framework and use suitable approximations of the unknowns. More specifically, we use Proper Generalized Decomposition (PGD) to solve the problems of the linear stage. This technique is presented in detail in Section 3.7

\subsection{The local stage at iteration $n+1$}

The local stage consists in building $\hat{\mathbf{s}}_{n+1 / 2} \in \boldsymbol{\Gamma}$ based on one's knowledge of $\mathbf{s}_{n} \in \mathbf{A}_{\mathbf{d}}$ using a search direction $\mathbf{E}^{+}$defined by:

$$
\mathbf{E}^{+} \forall \Omega_{E} \subset \Omega \quad\left\{\begin{array}{cc}
\left(\hat{\dot{\hat{\mathbf{e}}}}_{\mathrm{p}}-\dot{\mathbf{e}}_{\mathrm{p} E}\right)+\mathbf{H}+\left(\hat{\mathbf{f}}_{E}-\mathbf{f}_{E}\right) & =0 \\
\left(\underline{\dot{\hat{W}}}_{E}-\underline{\hat{W}}_{E}\right)-\mathbf{h}^{+}\left(\underline{\hat{F}}_{E}-\underline{F}_{E}\right) & =0
\end{array}\right.
$$

where $\mathbf{H}^{+}$and $\mathbf{h}^{+}$are symmetric positive operators which are parameters of the method. For the sake of simplicity, $\left(\mathbf{H}^{+}\right)^{-1}$ is often chosen equal to zero, which requires that the dual variables in the linear stage and the local stage be equal. $\mathbf{h}^{+}$can be viewed as the interface stiffness divided by a characteristic time. A simple value is: $\mathbf{h}^{+}=\frac{L}{E T} \mathbf{I}$, where $E$ is the Young's modulus, $T$ the duration of the phenomenon being studied, $L$ the largest dimension of the structure, and $\mathbf{I}$ the identity operator. At this stage, the problems are possibly nonlinear, but local in space (provided $\mathbf{B}$ is also local) and often also in time, so they lend themselves to the highest degree of parallelism.

\subsection{The linear stage at iteration $n+1$}

This stage consists in building $\mathbf{s}_{n+1} \in \mathbf{A}_{\mathbf{d}}$ based on one's knowledge of $\hat{\mathbf{s}}_{n+1 / 2} \in$ $\boldsymbol{\Gamma}$ using search direction $\mathbf{E}^{-}$. The substructure part of the search direction is defined by:

$$
\forall \Omega_{E} \subset \Omega \quad\left(\dot{\mathbf{e}}_{\mathrm{p} E}-\hat{\dot{\mathbf{e}}}_{\mathrm{p} E}\right)-\mathbf{H}^{-}\left(\mathbf{f}_{E}-\hat{\mathbf{f}}_{E}\right)=0
$$

Because of the admissibility of the macroforces, the boundary part of the search direction is obtained by introducing a Lagrange multiplier $\underline{\widetilde{W}}_{E}^{M}$ to guarantee the admissibility of the macroforces in a weak sense: $\underline{\tilde{W}}^{M}=\left(\underline{\widetilde{W}}_{E}^{M}\right)_{\Omega_{E} \subset \Omega} \in \mathcal{W}_{\text {ad }}^{M \star}$ :

$$
\forall \Omega_{E} \subset \Omega \quad\left(\underline{\dot{W}}_{E}-\underline{\hat{\dot{W}}}_{E}\right)+\mathbf{h}^{-}\left(\underline{F}_{E}-\underline{\hat{F}}_{E}\right)-\underline{\tilde{W}}_{E}^{M}=0
$$


Equations [19] and [20] define the search direction $\mathbf{E}^{-}$, where $\mathbf{h}^{-}=\mathbf{h}^{+}$, and a usual choice for $\mathbf{H}^{-}$is the (exact or approximate) tangential stiffness operator. The explicit expression of $\mathbf{H}^{-}$for a simple case is given in Equation [46.

The admissibility of the macroforces is expressed by:

$$
\begin{aligned}
& \forall \underline{\tilde{W}}_{E}^{M} \in \mathcal{W}_{\mathbf{a d}}^{M \star}, \\
& \sum_{\Omega_{E} \subset \Omega}\left\{\int_{I_{i}^{C} \times \partial \Omega_{E}} \dot{\widetilde{W}}_{E}^{M} \cdot \underline{F}_{E} d S d t-\int_{I_{i}^{C} \times \partial \Omega_{E} \cap \partial_{2} \Omega} \dot{\widetilde{W}}_{E}^{M} \cdot \underline{F}_{d} d S d t\right\}=0
\end{aligned}
$$

The search direction can be written in the form of a corrective term to the solution $\Delta \mathbf{s}_{n+1}=\mathbf{s}_{n+1}-\mathbf{s}_{n} \in \mathbf{A}_{\mathbf{d}}^{\star}$ :

$$
\begin{aligned}
& \Delta \dot{\mathbf{e}}_{\mathrm{p} E, n+1}-\mathbf{H}^{-} \Delta \mathbf{f}_{E, n+1}+\bar{\Delta}_{E, n+1}=0 \\
& \Delta \underline{\dot{W}}_{E, n+1}-\mathbf{h}^{-} \Delta \underline{F}_{E, n+1}+\underline{\bar{\delta}}_{E, n+1}=0
\end{aligned}
$$

with the following known quantities:

$$
\begin{aligned}
& \bar{\Delta}_{E, n+1}=\mathbf{H}^{-}\left(\hat{\mathbf{f}}_{E, n+1 / 2}-\mathbf{f}_{E, n}\right)-\left(\hat{\dot{\mathbf{e}}}_{\mathrm{p} E, n+1 / 2}-\dot{\mathbf{e}}_{\mathrm{p} E, n}\right) \\
& \underline{\bar{\delta}}_{E, n+1}=\mathbf{h}^{-}\left(\underline{\hat{F}}_{E, n+1 / 2}-\underline{F}_{E, n}\right)+\left(\underline{\hat{\dot{W}}}_{E, n+1 / 2}-\underline{\dot{W}}_{E, n}\right)+\underline{\dot{W}}_{E}^{M}
\end{aligned}
$$

For each $I_{i}^{C} \times \Omega_{E}$, the search direction can be interpreted as a linear constitutive relation. Thus, the linear stage consists in minimizing a global constitutive relation error in $\mathbf{A}_{\mathrm{d}}^{\star}$, which is defined over the space-time substructure $I_{i}^{C} \times \Omega_{E}$. In other words, it consists in finding $\Delta \mathbf{s}_{E, n+1}$ which minimizes, over $\mathbf{A}_{\mathbf{d}}^{\star}$, the constitutive relation error $e_{C R, E}^{2}\left(\Delta \mathbf{s}_{E, n+1}\right)$ associated with the search direction and defined by (omitting subscript $n+1$ ):

$$
e_{C R, E}^{2}\left(\Delta \mathbf{s}_{E}\right)=\left\|\Delta \dot{\mathbf{e}}_{\mathrm{p} E}-\mathbf{H}^{-} \Delta \mathbf{f}_{E}+\bar{\Delta}\right\|_{\mathbf{M}, E}^{2}+\left\|\Delta \underline{\dot{W}}_{E}+\mathbf{h}^{-} \underline{F}_{E}-\underline{\bar{\delta}}\right\|_{\mathbf{m}, E}^{2}
$$

where the corresponding norms are:

$$
\|\square\|_{\mathbf{M}, E}^{2}=\int_{I_{i}^{C} \times \Omega_{E}} \square \circ \mathbf{M} \square d \Omega d t \quad\|\square\|_{\mathbf{m}, E}^{2}=\int_{I_{i}^{C} \times \partial \Omega_{E}} \square \cdot \mathbf{m} \square d S d t
$$

In order to define a norm, operators $\mathbf{M}$ and $\mathbf{m}$ must be positive definite. For example, one can choose, if possible, $\mathbf{M}=\left(1-\frac{t}{T}\right)\left(\mathbf{H}^{-}\right)^{-1}$ and $\mathbf{m}=\left(1-\frac{t}{T}\right)\left(\mathbf{h}^{-}\right)^{-1}$, where $T$ is the duration of the problem being studied.

This problem depends on the quantities of the previous local stage $\hat{\mathbf{s}}_{E}$, on the known prescribed quantities $f_{d}$, on the initial conditions and on $\underline{\tilde{W}}_{E}^{M}$, which is unknown at this stage. Thanks to the linearity of the operator of the minimization problem, the solution can be viewed as the sum:

$$
\mathbf{s}_{E}=\tilde{\mathbf{s}}_{E}\left(\dot{\overline{\mathscr{W}}}_{E}^{M}\right)+\hat{\hat{\mathbf{s}}}_{E}
$$


where $\hat{\mathbf{s}}_{E}$ is the solution when $\underline{\tilde{W}}_{E}^{M}=0$. This solution can be calculated because it depends on fields which are known at this stage. The solution $\tilde{\mathbf{s}}_{E}$ depends linearly on $\underline{\tilde{W}}_{E}^{M}$, which is unknown. In particular, the previous relation also applies to the macroforces:

$$
\underline{F}_{E}^{M}=\mathbf{L}_{E} \underline{\tilde{W}}_{E}^{M}+\underline{\hat{\hat{F}}}_{E}^{M}
$$

where $\underline{\hat{\hat{F}}}_{E}^{M}$ is the macro part of the loading of $\hat{\hat{\mathbf{s}}}_{E}$, and $\mathbf{L}_{E}$ is a linear operator. $\mathbf{L}_{E}$ represents a condensation operator onto the coarse scale of the interfaces for Substructure $\Omega_{E}$ of Problem [23]. This operator is calculated for each substructure, which requires the resolution of a set of microproblems with zero initial conditions in which $\hat{\mathbf{s}}_{E}$ is equal to zero and $\underline{\tilde{W}}_{E}^{M}$ corresponds to each of the basis vectors of $\mathcal{W}_{E}^{M}$ in turn. The result at the interface is projected onto the coarse scale to obtain the corresponding value of $\underline{F}_{E}^{M}$ (the macro part of $\underline{F}_{E}$ ). This operator depends only on the choice of the macrobasis and on the parameters of the search directions $\mathbf{h}^{-}$and $\mathbf{H}^{-}$(for details, see Algorithm 4). Contrary to the evolution law of the viscoelastic material formulation, the evolution law of elastic-viscoplastic material formulation is not linear. The parameters of the search directions (linked to the tangential stiffness operator) are then not constant along the iterations of the LATIN method. As the operator $\mathbf{L}_{E}$ depends on the parameters of the search directions, it has to be computed at each update of the search directions. The computation cost of all the operators can be excessive even if the macroquantities belong to a small finite-dimension space. To reduce it, we choose to compute this operator only for the first iterations of the LATIN method, keeping it then constant even if the parameters of the search directions change. The convergence rate is not strongly affected by that simplification.

The macroproblem, whose solution is $\underline{\bar{W}}_{E}^{M} \in \mathcal{W}_{\text {ad }}^{M}$, is constructed by introducing the homogenized operator [26] into [21]:

$$
\begin{aligned}
\forall \underline{\widetilde{W}}_{E}^{M} & \in \mathcal{W}_{\mathbf{a d}}^{M \star}, \\
& \sum_{\Omega_{E} \subset \Omega}\left\{\int_{I_{i}^{C} \times \partial \Omega_{E}} \underline{\tilde{W}}_{E}^{\star}{ }^{\star} \cdot \mathbf{L}_{E} \dot{\widetilde{W}}_{E}^{M}-\dot{\widetilde{W}}_{E}^{M} \cdot\left(\underline{F}_{d}-\underline{\hat{F}}_{E}^{M}\right) d S d t\right\}=0
\end{aligned}
$$

This linear space-time problem is defined over the whole set of interfaces and the entire coarse subinterval $I_{i}^{C}$. One can prove that if the boundary conditions are taken into account through an interface behavior this problem has a unique solution. The macroproblem leads to $\underline{\tilde{W}}_{E}^{M}$ and then, through a microresolution, to $\tilde{\mathbf{s}}$. Thus, one can determine s completely. Therefore, the linear stage consists of four steps:

- minimization of [23] with $\underline{\tilde{W}}_{E}^{M}=\underline{0}$ (called "Microproblem 1"),

- if $\mathbf{H}^{-}$and/or $\mathbf{h}^{-}$and/or the macrobasis have changed, determination of operator $\mathbf{L}_{E}$, 
- $\underline{\hat{\hat{F}}}_{E}^{M}$ being now known, resolution of Macroproblem [27], leading to $\underline{\tilde{W}}_{E}^{M}$,

- minimization of [23] using $\hat{\mathbf{s}}=0$ and $\underline{\vec{W}}_{E}^{M}$ obtained previously (called "Microproblem 2").

\subsection{The proper generalized decomposition technique (PGD)}

This approximation, initially introduced in (Ladevèze, 1985) under the name "radial approximation", is part of the LATIN method. Previous works showed that it can lead to drastic reductions in computation costs. The basic idea is to approximate a function $f$ defined over a space-time domain $\Omega \times I_{i}^{G}$ as a sum of products of separated variable functions: a temporal function $\lambda_{i}(t)$ and a spatial function $\Lambda_{i}(\underline{M})$ : $\forall(t, \underline{M}) \in I_{i}^{C} \times \Omega$,

$$
f(t, \underline{M}) \approx \check{f}(t, \underline{M})=\sum_{j=1}^{m} \lambda_{j}(t) \Lambda_{j}(\underline{M})
$$

where the products $\lambda_{j}(t) \Lambda_{j}(\underline{M})$ are called "radial space-time functions". The number of products $m$ is not defined a priori since products are added in the course of the iterative method in order to ensure a good approximation in the sense of $e_{C R, E}^{2}$.

\subsubsection{The microproblem over $I_{i}^{C} \times \Omega_{E}$ at Iteration $n+1$, using $P G D$}

In (Ladevèze et al., 2010), it was shown that the minimum set of unknowns to be sought using a separated representation consists of the plastic deformation $\Delta \varepsilon_{\mathrm{p}}$, the interface displacement $\Delta \underline{W}$.

Let us assume that this set of unknowns is approximated through the adjunction of $k$ products of time functions by space functions. Dropping the subscript $\mathrm{E}$ for the sake of simplicity, this leads to:

$$
\begin{gathered}
\Delta \varepsilon_{\mathrm{p}}(t, \underline{M}) \approx \Delta \check{\varepsilon}_{\mathrm{p}}(t, \underline{M})=\sum_{j=1}^{k} \alpha_{j}^{p}(t) \mathbf{E}_{\mathbf{p}}^{j}(\underline{M}) \\
\Delta \underline{W}(t, \underline{M}) \approx \Delta \underline{\underline{W}}(t, \underline{M})=\sum_{j=1}^{k} \alpha_{j}^{W}(t) \underline{Z}^{j}(\underline{M})
\end{gathered}
$$

The same time function can be used for these quantities, i.e. $\alpha_{j}^{p}(t)=\alpha_{j}^{W}(t)$. This is possible because of the link done by the equilibrium equation. Let demonstrates it in the case of one pair, dropping indices $j$ for the sake of simplicity. The strain and the displacement are linked by a linear operator, and the interface displacement $\underline{W}$ is the projection on the interface of the internal displacement. All these quantities can have the same time function $\alpha^{u}=\alpha^{W}$,

$$
\Delta \underline{U}=\alpha^{u} \mathbf{U}
$$




$$
\begin{aligned}
& \Delta \varepsilon(\underline{U})=\alpha^{u} \nabla_{\text {sym }}(\mathbf{U})=\alpha^{u} \mathbf{E} \\
& \Delta \underline{W}=\alpha^{u} \underline{Z}
\end{aligned}
$$

As a correction to the elastic solution is searched, all $\Delta$ quantities are in $\mathbf{A}_{\mathrm{d}}^{\star}$. Introducing the state law and the partition of strain $\Delta \sigma=\mathbf{C}\left(\Delta \varepsilon-\Delta \varepsilon_{\mathrm{p}}\right)$ into the static admissibility conditions (Equation [15]) leads to $\forall \underline{U}^{\star} \in \mathcal{U}^{\star}$ and $\left.\underline{U}^{\star}\right|_{\Gamma}=0$,

$$
\int_{I_{i}^{C} \times \Omega} \mathbf{C}\left(\Delta \varepsilon-\Delta \varepsilon_{\mathrm{p}}\right): \varepsilon\left(\underline{\dot{U}}^{\star}\right) d \Omega d t=0
$$

Introducing the PGD, the test field becomes $\underline{U^{\star}}=\alpha^{u \star} \mathbf{U}+\alpha^{u} \mathbf{U}^{\star}$, with $\mathbf{U}^{\star} \in \mathcal{U}^{\star}$ and no condition is required on $\alpha^{u \star}$. Because there is no condition on $\alpha^{u \star}$, a space problem and a time problem can be separated. The space problem consists in seeking $\mathbf{U} \in \mathcal{U}^{2}=\left\{\mathbf{U} \in \mathcal{U}_{E}^{\star}|\mathbf{U}|_{\Gamma}=\underline{Z}\right\}$ such that $\forall \mathbf{U}^{\star} \in \mathcal{U}^{\star}$,

$$
\int_{I_{i}^{C}} \alpha^{u} \alpha^{u} d t \int_{\Omega} \mathbf{C} \boldsymbol{\varepsilon}(\mathbf{U}): \varepsilon\left(\mathbf{U}^{\star}\right) d \Omega=\int_{I_{i}^{C}} \alpha^{u} \alpha^{p} \int_{\Omega} \mathbf{C E} \mathbf{E}_{\mathbf{p}}: \varepsilon\left(\mathbf{U}^{\star}\right) d \Omega
$$

The time problem consists in finding $\alpha^{u}$ such that $\forall \alpha^{u \star}$,

$$
\int_{I_{i}^{C}} \alpha^{u} \alpha^{u \star} d t \int_{\Omega} \mathbf{C E}: \varepsilon(\mathbf{U}) d \Omega=\int_{I_{i}^{C}} \alpha^{p} \alpha^{u \star} \int_{\Omega} \mathbf{C E}_{\mathbf{p}}: \varepsilon(\mathbf{U}) d \Omega
$$

From the time Problem [32], one can deduce that $\alpha^{u}$ and $\alpha^{p}$ are proportional. We make the choice of having $\alpha^{u}=\alpha^{p}$ which sets the value of $\mathbf{E}$ knowing $\mathbf{E}_{\mathbf{p}}$. All the time functions are the same and are then denoted $\alpha=\alpha^{p}=\alpha^{u}=\alpha^{W}$.

With this choice for the time functions, the spatial functions $\mathbf{E}$ is obtained through the resolution of the space Problem [31] which consists in seeking $\mathbf{U} \in \mathcal{U}^{2}$ such that

$$
\forall \mathbf{U}^{\star} \in \mathcal{U}^{\star} \quad \int_{\Omega} \mathbf{C}\left(\varepsilon(\mathbf{U})-\mathbf{E}_{\mathbf{p}}\right): \varepsilon\left(\mathbf{U}^{\star}\right) d \Omega=0
$$

Then, the strain is deduced through $\mathbf{E}=\nabla_{\text {sym }}(\mathbf{U})$ and the stress $\Delta \boldsymbol{\sigma}=\alpha \mathbf{S}$ is given by:

$$
\mathbf{S}=\mathbf{C}\left(\mathbf{E}-\mathbf{E}_{\mathbf{p}}\right)
$$

Finally, the interface forces $\Delta \underline{F}=\alpha \underline{G}$ are obtained with:

$$
\underline{G}=\left.\mathbf{S} \underline{n}\right|_{\partial \Omega_{E}}
$$

One must notes that the solution is searched as a correction to the elastic solution. Then, if no pairs are added $(k=0)$ the quantities have their elastic values. In the case of an elastic substructure in a plastic problem, the only quantities to correct are the interfaces displacements and forces. Pairs are added in that case, which combination gives zero plastic strain. The principle of adding one product in order to improve the quality of the approximation consists in seeking a minimum of $e_{R C, E}^{2}\left(\Delta \mathbf{s}_{E}\right)$ [23] with 


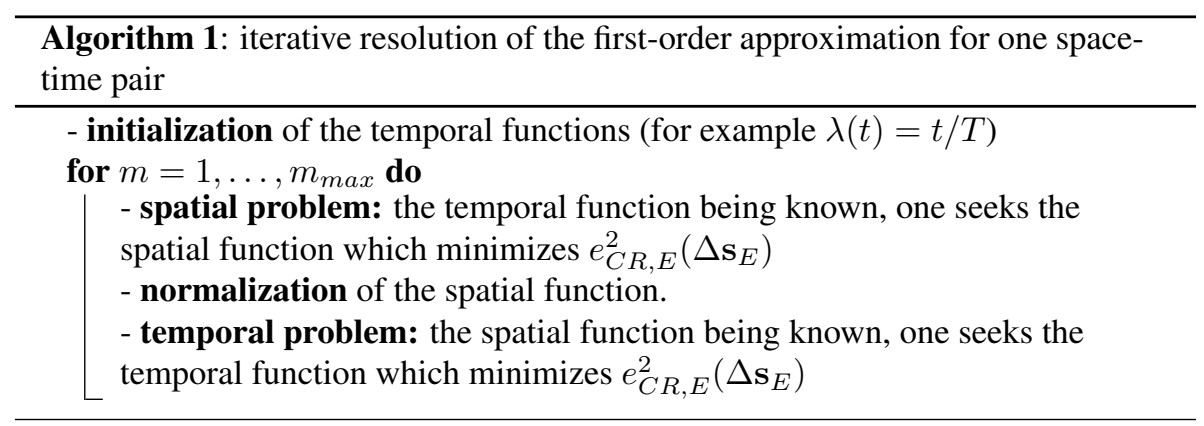

respect to time and space alternatively using Algorithm 1 The minimization with respect to the time variable leads to a scalar differential equation defined over $I_{i}^{C}$, whose resolution is quite inexpensive. The minimization with respect to the spatial variable leads to the resolution of a small number of classical time-independent finite element problems defined over $\Omega_{E}$. Both the spatial and the temporal problems are linear problems, provided the search direction and the norm operator are linear operators. As can be seen in our examples, Algorithm 1 converges very quickly. Therefore, in practice, $m_{\max }$ is chosen to be equal to only 2 or 3 .

\subsubsection{Practical use of this technique in the strategy}

Since the construction of the spatial functions is, by far, the most expensive part of the process, it is worth storing and reusing these functions (see (Nouy et al., 2004; Ladevèze et al., 2010)). Thus, the spatial functions constructed up to Iteration $n$ are reused systematically at Iteration $n+1$ (Algorithm 2). In a first step, called the "use of the reduced basis", only the temporal functions are updated in order to minimize the constitutive relation error. An error indicator $\xi_{C R, E}$ which quantifies the accuracy of this first prediction is calculated. For each quantity being considered, if the error is less than a critical value $\xi^{0}$ then the quantity is considered to be well-described; no other operation is performed and one proceeds to the next iteration. Otherwise, new space-time pairs are sought. One can observe that Parameter $\xi^{0}$ affects either the convergence rate (if $\xi^{0}$ is too large) or the computation cost (if $\xi^{0}$ is too small). Numerical tests show that a good compromise is obtained with $\xi^{0}=0.3$.

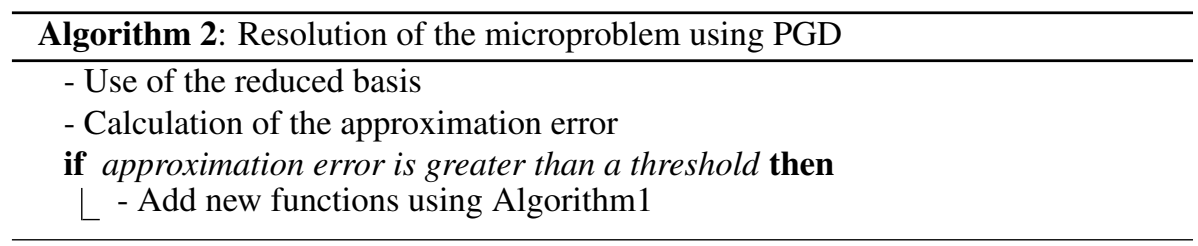




\subsection{Convergence of the algorithm}

The convergence of the LATIN method was proven in the case of a standard constitutive law (associated potential). If one assumes that the constitutive relation operator $\mathbf{B}$ is monotonous, that the interfaces are perfect and that the search direction operators $\mathbf{H}^{-}, \mathbf{H}+$ are positive definite (see (Ladevèze, 1999)), $\frac{1}{2}\left(\mathbf{s}_{n}+\mathbf{s}_{n+1}\right)$ converges toward the solution sexact.

Since the solution $\mathbf{S}_{\text {exact }}$ is the intersection of $\mathbf{A}_{\mathbf{d}}$ and $\boldsymbol{\Gamma}$, a measure of the distance between $\hat{\mathbf{s}}_{n+1 / 2}$ and $\mathbf{s}_{n+1}$ is a good error indicator to verify the convergence of the strategy. For example, one can use:

$$
\eta^{2}=\frac{\left\|\hat{\mathbf{s}}_{n+1 / 2}-\mathbf{s}_{n+1}\right\|^{2}}{\left\|\hat{\mathbf{s}}_{n+1 / 2}+\mathbf{s}_{n+1}\right\|^{2}}
$$

with:

$$
\begin{aligned}
\|\mathbf{s}\|^{2}= & \frac{1}{2} \sum_{\Omega_{E}} \sum_{I_{i}^{C}} \int_{\Omega_{E} \times I_{i}^{C}}\left(\mathbf{f}_{E} \circ \mathbf{H}^{-} \mathbf{f}_{E}+\dot{\mathbf{e}}_{\mathrm{p} E} \circ\left(\mathbf{H}^{-}\right)^{-1} \dot{\mathbf{e}}_{\mathrm{p} E}\right) d \Omega d t+ \\
& \int_{\partial \Omega_{E} \times I_{i}^{C}}\left(\underline{F}_{E} \cdot \mathbf{h}^{-} \underline{F}_{E}+\underline{\dot{W}}_{E} \cdot\left(\mathbf{h}^{-}\right)^{-1} \underline{\dot{W}}_{E}\right) d S d t
\end{aligned}
$$

Classically, in order to ensure convergence of the algorithm for more general types of behavior, one modifies the linear stage by adding a relaxation step: one renames the quantity previously denoted $\mathbf{s}_{n+1}$ to $\breve{\mathbf{s}}_{n+1}$ and defines $\mathbf{s}_{n+1}$ using the relation: $\mathbf{s}_{n+1}=\mu \breve{\mathbf{s}}_{n+1}+(1-\mu) \mathbf{s}_{n}$ where $\mu$ is a relaxation parameter usually chosen to be equal to 0.8 . This value is a parameter of the method. The convergence of the method is proven for $\mu=0.5$, but a better convergence rate can be obtained using $\mu$ closer to one. According to some tests, the value 0.8 leads to a good convergence rate.

\subsection{A word concerning the use of different functional spaces}

One can observe that the exchange of data between two substructures is carried out only at their interface. This property enables one to deal with different functional spaces in the substructures in terms of PGD approximation and/or discretization schemes. Before solving the behavior equations of the interfaces, a necessary first step consists in building the trace of all the PGD quantities at the interfaces. Then, if the discretization schemes at the substructure boundaries are the same, classically, the interface behavior is used. If they are different, a particular technique such as the mortar-based transfer technique introduced in (Néron et al., 2008) must be used. 


\section{Example of a bar}

\subsection{Definition of the problem}

Let us consider the academic example of a bar in traction subjected to quasistatic isothermal evolution, with the assumption of small perturbations. The material's behavior is elastic-viscoplastic and is described by the normal formulation of the Marquis-Chaboche constitutive law (see Section 2.2). The bar is subjected to a zero displacement at $x=0$ and to a prescribed displacement $U=U_{d}$ at the other end $x=L$. The structure is made of three different materials in the three parts denoted 1,2,3 on Figure 3 . The corresponding material coefficients are given in Table 1 . The material are distinguished by the initial threshold $\sigma_{y}$, as it is one of the most influential parameter on the plastic strain level.

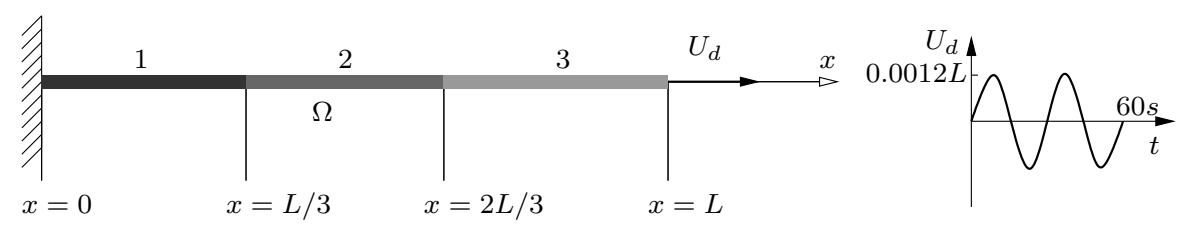

Figure 3. Definition of the one-dimensional example

Table 1. Elastic-viscoplastic constitutive coefficients for a 316 Steel at $600^{\circ} \mathrm{K} . \mathrm{E}, \mathcal{C}$, refer to Equation [6]. $\sigma_{y}$ refers to Equation [8]. $K, \mathcal{N}$ and $\gamma$ refers to [10] and [11]

\begin{tabular}{cccccccc}
\hline part & $E$ & $\nu$ & $\mathcal{C}$ & $\sigma_{y}$ & $\mathcal{N}$ & $K$ & $\gamma$ \\
\hline 1 & $137,600 \mathrm{Mpa}$ & 0.3 & $24,800 \mathrm{Mpa}$ & $8 \mathrm{Mpa}$ & 12 & $150 \mathrm{MPa} . s^{1 / \mathcal{N}}$ & 300 \\
2 & $"$ & $"$ & $"$ & $6 \mathrm{Mpa}$ & $"$ & $"$ & $"$ \\
3 & $"$ & $"$ & $"$ & $4 \mathrm{Mpa}$ & $"$ & $"$ & $"$ \\
\hline
\end{tabular}

\subsection{Substructuring of the problem}

The structure is viewed as an assembly of substructures and interfaces (Figure 4). An interface between two substructures has four unknowns (the displacement and the force at each end). Each boundary interface has two unknowns, the displacement and the force at the end connected to the substructure. One can observe that the substructure boundaries do not need to coincide with the change of material.

The macroquantities at the interfaces are defined as projections onto the macrobasis, which consists of three space-time functions (see Figure 5) defined on a single coarse time interval $\left(I=I_{1}^{C}=[0, T]\right)$. This basis can be enriched automatically during the calculation to ensure scalability when dealing with highly nonlinear problems, as explained in (Passieux et al., 2010). 


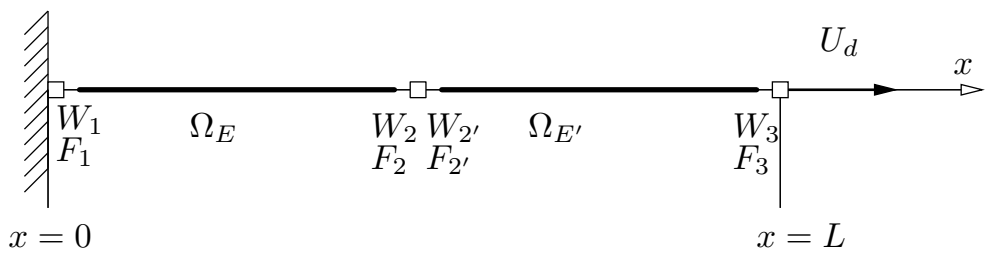

Figure 4. Substructuring of the problem

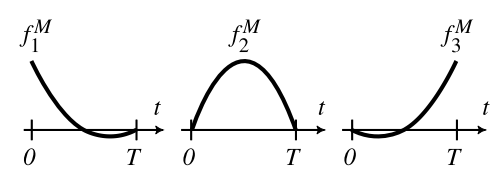

Figure 5. The space-time macrobasis

\subsection{Definition of the spaces}

In this one-dimensional case, spaces $\mathbf{A}_{\mathbf{d}}$ and $\boldsymbol{\Gamma}$ are defined as:

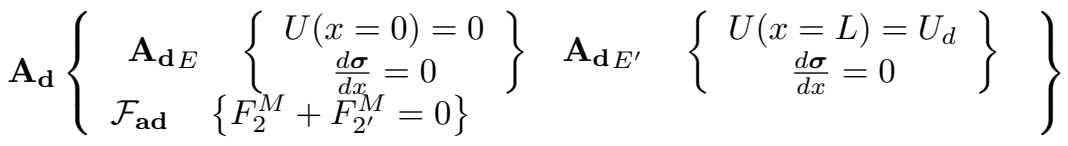

$$
\begin{aligned}
& \boldsymbol{\Gamma}\left\{\begin{array}{l}
\dot{\mathbf{e}}_{\mathrm{p}}=\mathbf{B}(\mathbf{f}) \\
W_{2}=W_{2^{\prime}} \quad F_{2}+F_{2^{\prime}}=0
\end{array}\right\}
\end{aligned}
$$

The solution is the intersection $\mathbf{s}_{\text {exact }}=\mathbf{A}_{\mathbf{d}} \cap \boldsymbol{\Gamma}$ of these two spaces and can be found using the LATIN iterative procedure.

\subsection{Discretization schemes}

In each substructure, the spatial discretization is carried out using a classical finite element scheme consisting, for example, of four linear elements as shown in Figure 6 A derivation operator $B$ is associated with that discretization, and a Gaussian integration scheme is used. The fields of the spatial derivative fields are constant over each element. 


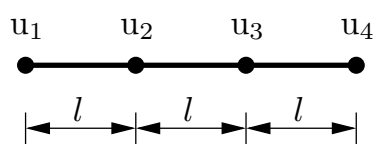

Figure 6. The spatial discretization of a substructure

A Euler scheme is used for the time discretization, so the time derivative of a function $\alpha$ at time step $i$ is:

$$
\dot{\alpha}_{i}=\frac{\alpha_{i}-\alpha_{i-1}}{\delta t}
$$

In our case, sixty time steps were used.

\subsection{The local stage at iteration $n+1$}

Given a solution $\mathbf{s}_{n} \in \mathbf{A}_{\mathbf{d}}$, one seeks $\hat{\mathbf{s}}_{n+1 / 2} \in \boldsymbol{\Gamma}$. The most natural substructure search direction (Equation [18]) to choose is given by $(\mathbf{H}+)^{-1}=0$ i.e. $\hat{\mathbf{f}}=\mathbf{f}$. With this search direction, the local stage in the substructures consists in finding $\hat{\dot{\mathbf{e}}}_{n+1 / 2}$ such that

$$
\begin{aligned}
\hat{\mathbf{e}}_{n+1 / 2}=\mathbf{B}\left(\mathbf{f}_{n}\right) \Leftrightarrow & \Leftrightarrow \\
& {\left[\begin{array}{c}
\hat{\dot{\varepsilon}}_{\mathrm{p} \mathrm{n}+1 / 2} \\
-\dot{\boldsymbol{\alpha}}_{n+1 / 2}
\end{array}\right]=k\langle f\rangle_{+}^{\mathcal{N}}\left[\begin{array}{c}
\operatorname{sign}\left(\boldsymbol{\sigma}_{n}-\boldsymbol{X}_{n}\right) \\
-\operatorname{sign}\left(\boldsymbol{\sigma}_{n}-\boldsymbol{X}_{n}\right)+\frac{3 \gamma}{2} \frac{1}{\mathcal{C}} \boldsymbol{X}_{n}
\end{array}\right] }
\end{aligned}
$$

with

$$
f=\left|\sigma_{n}-\boldsymbol{X}_{n}\right|-\sigma_{y}
$$

These two equations correspond to Equations [12] and [8] in the case of a bar.

At the interfaces, we choose to neglect the influence of viscosity and use the same search direction as in the linear case. This search direction is a function of only the neighboring substructures, with length $l$ and stiffness $\mathbf{C}$ given by the Young's modulus $E$, and with a characteristic time $t_{c}$ related to the loading (in this case, equal to the period of the loading):

$$
\mathbf{h}^{+}=\frac{1}{t_{c}} \frac{l}{E}
$$

On each side of the interface, the search directions are defined by:

$$
\left\{\begin{array}{l}
\left(\hat{\dot{W}}-\dot{W}_{n}\right)-\mathbf{h}\left(\hat{F}-F_{n}\right)=0 \\
\left(\hat{\dot{W}}^{\prime}-\dot{W}_{n}^{\prime}\right)-\mathbf{h}^{\prime}\left(\hat{F}^{\prime}-F_{n}^{\prime}\right)=0
\end{array}\right.
$$


with $\mathbf{h}=\mathbf{h}^{\prime}=\mathbf{h}^{+}$. Combining Equations [39] and [43], one has

$$
\left\{\begin{array}{l}
\hat{\dot{W}}=\hat{\dot{W}}^{\prime}=\left(\mathbf{h}^{-1}+\mathbf{h}^{\prime-1}\right)^{-1}\left\{\mathbf{h}^{-1} \dot{W}_{n}+\mathbf{h}^{\prime-1} \dot{W}_{n}^{\prime}-\left(F_{n}+F_{n}^{\prime}\right)\right\} \\
\hat{F}=-\hat{F}^{\prime}=F_{n}+\mathbf{h}^{-1}\left(\hat{\dot{W}}_{n}-\dot{W}_{n}\right)
\end{array}\right.
$$

For the interfaces with prescribed displacement, one has

$$
\left\{\begin{array}{l}
\hat{W}=U_{d} \\
\hat{F}=F_{n}+\mathbf{h}^{-1}\left(\dot{U}_{d}-\dot{W}_{n}\right)
\end{array}\right.
$$

\subsection{The linear stage at iteration $n+1$}

As explained in Section 3.6, the linear stage consists of two microproblems in the substructures and a macroproblem at the interfaces.

\subsubsection{The search direction (Equations [19] and [20])}

In the substructures, the search direction leading to the smallest number of iterations is the tangent to space $\boldsymbol{\Gamma}$ in $\hat{\mathbf{s}}_{n+1 / 2}$, i.e. the derivative of $\Phi(f) \partial_{\mathbf{f}} F$ with respect to $\mathbf{f}$ (see (Ladevèze, 1999)). Then, the linear time-dependent operator $\mathbf{H}^{-}$is equal to:

$$
\begin{aligned}
\mathbf{H}^{-}=k\langle f\rangle_{+}^{\mathcal{N}} & {\left[\begin{array}{cc}
0 & 0 \\
& \frac{3 \gamma}{2 \mathcal{C}}
\end{array}\right] } \\
& +k \mathcal{N}\langle f\rangle_{+}^{\mathcal{N}-1}\left[\begin{array}{cc}
1 & -1 \\
\operatorname{sign}(\boldsymbol{\sigma}-\boldsymbol{X}) C_{1} & -\operatorname{sign}(\boldsymbol{\sigma}-\boldsymbol{X}) C_{1}
\end{array}\right]
\end{aligned}
$$

with

$$
C_{1}=\left(-\operatorname{sign}(\boldsymbol{\sigma}-\boldsymbol{X})+\frac{3 \gamma}{2 \mathcal{C}} \boldsymbol{X}\right) .
$$

On the one hand, the calculation of this search direction can be computationally expensive and requires the homogenized operator $\mathbf{L}$ to be updated. On the other hand, keeping the same search direction for too many iterations affects the convergence rate of the algorithm. Updating the search direction only when the convergence rate slows down is an efficient strategy.

For the sake of simplicity, the off-diagonal terms are set to zero. This enables one to divide the constitutive relation error into two parts, one related to $\left(\varepsilon_{\mathrm{p}}, \underline{W}\right)$ and the other to $\boldsymbol{\alpha}$. Then, the search direction can be written as:

$$
\mathbf{H}^{-}=\left[\begin{array}{cc}
\mathbf{H}_{\boldsymbol{\sigma}} & 0 \\
0 & \mathbf{H}_{\mathbf{X}}
\end{array}\right]
$$

with

$$
\mathbf{H}_{\boldsymbol{\sigma}}=k \mathcal{N}\langle f\rangle_{+}^{\mathcal{N}-1}, \quad \mathbf{H}_{\mathbf{X}}=k\langle f\rangle_{+}^{\mathcal{N}} \frac{3 \gamma}{2 \mathcal{C}}-k \mathcal{N}\langle f\rangle^{\mathcal{N}-1} \operatorname{sign}(\boldsymbol{\sigma}-\boldsymbol{X}) C_{1}
$$


The search direction derives from the evolution law and is a positive operator, but is not definite. Indeed, in the elastic domain, $f=0$ and $\mathbf{H}^{-}$is zero. In order to take $\mathbf{M}=\left(\mathbf{H}^{-}\right)^{-1}$, one introduces a minimum positive operator $\mathbf{Z}$ such that

$$
\mathbf{H}^{-}=\mathbf{H}^{-}+\mathbf{Z}
$$

with, for example, $\mathbf{Z}=\zeta \mathbf{A}^{-1}$, where $\zeta$ denotes a regularization coefficient to be optimized, and $\mathbf{A}$ is the state law operator. $\zeta$ must be as small as possible. In our test, we found the value 0.15 to be the minimum. The search direction of the interface at the linear stage $\mathbf{h}^{-}$is chosen to be equal to that of the local stage $\mathbf{h}^{+}$.

\subsubsection{Resolution of the microproblems using PGD, plastic deformation part}

Algorithm 1 enables one to determine a new pair of functions in the PGD approximation. In this section, we give a detailed description of the different minimization problems for the chosen discretization scheme. The constitutive error is:

$$
e_{C R, E}^{2}\left(\Delta \mathbf{s}_{E, n}\right)=e_{C R \varepsilon_{\mathrm{p}}, E}^{2}\left(\Delta \varepsilon_{\mathrm{p}}, \Delta W\right)
$$

which corresponds to Equation [23] in the case of a bar. The correction is sought in PGD form, i.e. as the product of a spatial function and a temporal function:

$$
e_{C R \varepsilon_{\mathrm{p}}, E}^{2}\left(\Delta \check{\varepsilon}_{\mathrm{p}}, \Delta \check{W}\right)=\left\|\dot{\alpha} \mathbf{E}_{p}-\mathbf{H}_{\boldsymbol{\sigma}} \alpha \mathbf{S}+\bar{\Delta}_{\boldsymbol{\varepsilon}}\right\|_{\mathbf{M}_{\boldsymbol{\sigma}}}+\left\|\dot{\alpha} Z+\mathbf{h}^{-} \alpha G-\bar{\delta}\right\|_{\mathbf{m}}
$$

where $\mathbf{M}_{\boldsymbol{\sigma}}$ is the part of $\mathbf{M}$ related to $\varepsilon$.

In this case, the resolution of a microproblem consists in minimizing the error, given $\left(\bar{\Delta}_{\varepsilon_{\mathrm{p}}}, \bar{\delta}\right)$.

Adjunction of a new pair to represent $\left(\Delta \varepsilon_{\mathrm{p}}, \Delta \underline{W}\right)=\left(\alpha(t) \mathbf{E}_{\mathbf{p}}, \alpha(t) \underline{Z}\right)$

- The spatial problem leading to $\left(\mathbf{E}_{p}, \underline{Z}\right)$

Given the temporal function $\alpha(t)$, one seeks $\left(\mathbf{E}_{p}, Z\right)$ which minimizes $e_{C R, E}^{2}\left(\Delta \mathbf{s}_{E}\right)$. With that formulation, one needs to solve a finite element problem with twice the size of a standard problem. Another way of finding the minimum consists in seeking the unknowns in a different order. Introducing the kinematic unknown $\Delta \check{\varepsilon}=\alpha \mathbf{E}$, the constitutive relation error can be written as:

$$
\begin{aligned}
e_{C R \varepsilon_{\mathrm{p}}, E}^{2}\left(\Delta \check{\varepsilon}_{\mathrm{p}}, \Delta \check{W}\right)=\| \dot{\alpha} \mathbf{C}^{-1} \mathbf{S}-\mathbf{H}_{\boldsymbol{\sigma}} \alpha \mathbf{S}- & \dot{\alpha} \mathbf{E}+\bar{\Delta}_{\boldsymbol{\varepsilon}} \|_{\mathbf{M}_{\boldsymbol{\sigma}}} \\
& +\left\|\dot{\alpha} Z+\mathbf{h}^{-} \alpha G-\bar{\delta}\right\|_{\mathbf{m}}
\end{aligned}
$$

Then, the stationarity of the error is expressed by: find $(\mathbf{E}, Z) \in \mathcal{E}_{E}^{\star}$ and $(\mathbf{S}, G) \in$ $\mathcal{F}_{E}^{\star}$ such that $\forall\left(\mathbf{E}^{\star}, Z^{\star}\right) \in \mathcal{E}_{E}^{\star}$ and $\forall\left(\mathbf{S}^{\star}, G^{\star}\right) \in \mathcal{F}_{E}^{\star}$

$$
\left\{\begin{array}{c}
\int_{\Omega_{E}} \mathbf{S}^{\star}:\left(A_{C C} \mathbf{S}-A_{C E} \mathbf{E}+\bar{\Delta}_{C}\right) d \Omega \\
+\int_{\partial \Omega_{E}} G^{\star} \cdot\left(a_{G G} G+a_{G Z} Z-\bar{\delta}_{G}\right) d \Gamma=0 \\
\int_{\Omega_{E}} \mathbf{E}^{\star}:\left(A_{E E} \mathbf{E}-A_{E C} \mathbf{S}-\bar{\Delta}_{E}\right) d \Omega \\
+\int_{\partial \Omega_{E}} Z^{\star} \cdot\left(a_{Z Z} Z+a_{Z G} G-\bar{\delta}_{Z}\right) d \Gamma=0
\end{array}\right.
$$


with

$$
\begin{aligned}
& \mathrm{A}_{C C}=\frac{1}{E^{2}} \sum_{t=0}^{T} \frac{1}{\mathbf{H}_{\sigma}} \dot{\alpha}^{2} d t+\frac{2}{E} \sum_{t=0}^{T} \dot{\alpha} \alpha d t+\sum_{t=0}^{T} \mathbf{H}_{\boldsymbol{\sigma}} \alpha^{2} d t \\
& \mathrm{~A}_{C E}=\mathrm{A}_{E C}=\frac{1}{E} \sum_{t=0}^{T} \frac{1}{\mathbf{H}_{\sigma}} \dot{\alpha}^{2} d t+\sum_{t=0}^{T} \dot{\alpha} \alpha \delta t \\
& \mathrm{~A}_{E E}=\sum_{t=0}^{T} \frac{1}{\mathbf{H}_{\sigma}} \dot{\alpha}^{2} \delta t \\
& \bar{\Delta}_{C}=-\sum_{t=0}^{T} \frac{\Delta_{\varepsilon}}{\Delta_{\varepsilon}} \delta t-\frac{1}{E} \sum_{t=0}^{T} \frac{1}{\mathbf{H}_{\sigma}} \bar{\delta} \dot{\alpha} \delta t \\
& \bar{\Delta}_{E}=-\sum_{t=0}^{T} \frac{1}{\mathbf{H}_{\sigma}} \bar{\Delta}_{\boldsymbol{\varepsilon}} \dot{\alpha} \delta t \\
& \mathrm{a}_{G G}=\sum_{t=0}^{T} \mathbf{h}^{-} \alpha^{2} \delta t \\
& \mathrm{a}_{G Z}=a_{Z G}=\sum_{t=0}^{T} \dot{\alpha} \alpha \delta \\
& \mathrm{a}_{Z Z}=\sum_{t=0}^{T} \frac{1}{\mathbf{h}^{-}} \dot{\alpha}^{2} \delta t \\
& \bar{\delta}_{G}=\sum_{t=0}^{T} \bar{\delta} \alpha \delta \\
& \bar{\delta}_{Z}=\sum_{t=0}^{T} \frac{1}{\mathbf{h}^{-}} \bar{\delta} \dot{\alpha} \delta t
\end{aligned}
$$

System [51] of two equations with two unknowns is global because of the admissibility conditions and is solved using Algorithm 3 .

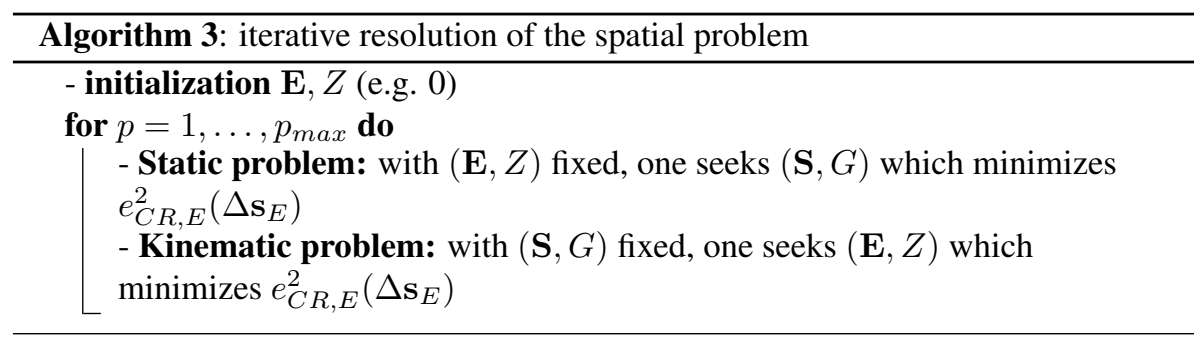

- The static problem: This linear problem can be converted to a primal problem through a duality transformation. Two Lagrange multipliers $(\tilde{\mathbf{E}}, \tilde{W})$ are introduced in order to ensure that $(\mathbf{S}, G)$ belongs to $\mathcal{F}^{\star}$, i.e.:

$$
\begin{aligned}
& \forall\left(\mathbf{S}^{\star}, G^{\star}\right) \quad \int_{\Omega_{E}} \mathbf{S}^{\star}:\left(A_{C C} \mathbf{S}-A_{C E} \mathbf{E}+\bar{\Delta}_{C}\right) d \Omega \\
& +\int_{\partial \Omega_{E}} G^{\star} \cdot\left(a_{G G} G+a_{G Z} Z-\bar{\delta}_{G}\right) d \Gamma-\int_{\Omega_{E}} \mathbf{S}^{\star}: \tilde{\mathbf{E}} d \Omega+\int_{\partial \Omega_{E}} G^{\star} \cdot \tilde{Z} d S=0 \\
& \forall\left(\tilde{\mathbf{E}}^{\star}, \tilde{\tilde{Z}}^{\star}\right) \in \mathcal{E}_{E}^{\star}, \quad \int_{\Omega_{E}} \mathbf{S}: \tilde{\mathbf{E}}^{\star}=\int_{\partial \Omega_{E}} G \cdot \tilde{Z}^{\star} d S
\end{aligned}
$$

Since no condition applies to $\left(\mathbf{S}^{\star}, G^{\star}\right)$, Equation [53] can be written at any point

$$
\begin{aligned}
& \mathbf{S}=A_{C C}^{-1}\left(A_{C E} \mathbf{E}-\bar{\Delta}_{C}+\tilde{\mathbf{E}}\right) \\
& G=a_{G G}^{-1}\left(\bar{\delta}_{G}-a_{G Z} Z-\tilde{Z}\right)
\end{aligned}
$$


These relations are introduced into Equation [54], the admissibility equation of the Lagrange multipliers:

$$
\begin{aligned}
& \int_{\Omega_{E}} \tilde{\mathbf{E}}^{\star}: A_{C C}^{-1}\left(A_{C E} \mathbf{E}-\bar{\Delta}_{C}+\tilde{\mathbf{E}}\right) d \Omega= \\
& \int_{\partial \Omega_{E}} \tilde{Z}^{\star} \cdot a_{G G}^{-1}\left(\bar{\delta}_{G}-a_{G Z} Z-\tilde{Z}\right) d S
\end{aligned}
$$

This problem can be solved as a classical finite element problem. Then, $(\mathbf{S}, \underline{G})$ can be calculated thanks to Equation [55].

$$
\begin{array}{r}
{\left[\begin{array}{cccc}
\frac{\mathrm{A}_{C C}^{-1}}{l}+\mathrm{a}_{G G}^{-1} & -\frac{\mathrm{A}_{C C}^{-1}}{l} & 0 & 0 \\
-\frac{\mathrm{A}_{C C}^{-1}}{l} & 2 \frac{\mathrm{A}_{C C}^{-1}}{l} & -\frac{\mathrm{A}_{C C}^{-1}}{l} & 0 \\
0 & -\frac{\mathrm{A}_{C C}^{-1}}{l} & 2 \frac{\mathrm{A}_{C C}^{-1}}{l} & -\frac{\mathrm{A}_{C C}^{-1}}{l} \\
0 & 0 & -\frac{\mathrm{A}_{C C}^{-1}}{l} & \frac{\mathrm{A}_{C C}^{-1}}{l}+\mathrm{a}_{G G}^{-1}
\end{array}\right]\left[\begin{array}{c}
\tilde{\mathrm{u}}_{1} \\
\tilde{\mathrm{u}}_{2} \\
\tilde{\mathrm{u}}_{3} \\
\tilde{\mathrm{u}}_{4}
\end{array}\right]=} \\
\\
{\left[\begin{array}{c}
\frac{\mathrm{A}_{C C}^{-1}}{l}\left(-\mathrm{A}_{C E} \mathrm{E}+\bar{\Delta}_{C}\right)+\mathrm{a}_{G G}^{-1}\left(\mathrm{a}_{G Z} Z-\bar{\delta}_{G}\right) \\
\frac{\mathrm{A}_{C C}^{-1}}{l}\left(-\mathrm{A}_{C E} \mathrm{E}+\bar{\Delta}_{C}\right) \\
\frac{\mathrm{A}_{C C}^{-1}}{l}\left(-\mathrm{A}_{C E} \mathrm{E}+\bar{\Delta}_{C}\right) \\
\frac{\mathrm{A}_{C C}^{-1}}{l}\left(-\mathrm{A}_{C E} \mathrm{E}+\bar{\Delta}_{C}\right)+\mathrm{a}_{G G}^{-1}\left(\mathrm{a}_{G Z} Z-\bar{\delta}_{G}\right)
\end{array}\right]}
\end{array}
$$

and the static unknown can be deduced:

$$
\begin{aligned}
& Z(1)=\tilde{\mathrm{u}}_{1} \quad Z(2)=\tilde{\mathrm{u}}_{4} \quad \tilde{\mathrm{E}}=B \tilde{\mathrm{u}} \\
& G=\mathrm{a}_{G G}^{-1}\left(\bar{\delta}_{G}-\mathrm{a}_{G Z} Z-\tilde{\mathrm{Z}}\right) \quad \mathrm{C}=\mathrm{A}_{C C}^{-1}\left(\mathrm{~A}_{C E} \mathrm{E}-\bar{\Delta}_{C}+\tilde{\mathrm{E}}\right)
\end{aligned}
$$

- The kinematic problem: The kinematic problem is already a primal problem which can be solved using the finite element method.

$$
\begin{aligned}
& {\left[\begin{array}{cccc}
\frac{\mathrm{A}_{E E}}{l}+\mathrm{a}_{Z Z} & -\frac{\mathrm{A}_{E E}^{-1}}{l} & 0 & 0 \\
-\frac{\mathrm{A}_{E E}}{l} & 2 \frac{\mathrm{A}_{E E}}{l} & -\frac{\mathrm{A}_{E E}}{l} & 0 \\
0 & -\frac{\mathrm{A}_{E E}}{l} & 2 \frac{\mathrm{A}_{E E}}{l} & -\frac{\mathrm{A}_{E E}}{l} \\
0 & 0 & -\frac{\mathrm{A}_{E E}}{l} & \frac{\mathrm{A}_{E E}}{l}+\mathrm{a}_{Z Z}
\end{array}\right]\left[\begin{array}{l}
\mathrm{u}_{1} \\
\mathrm{u}_{2} \\
\mathrm{u}_{3} \\
\mathrm{u}_{4}
\end{array}\right]=} \\
& \\
& \\
& {\left[\begin{array}{c}
\frac{1}{l}\left(\mathrm{~A}_{E C} \mathrm{C}-\bar{\Delta}_{E}\right)+\left(\mathrm{a}_{Z G} G+\bar{\delta}_{Z}\right) \\
\frac{1}{l}\left(\mathrm{~A}_{E C} \mathrm{C}-\bar{\Delta}_{E}\right) \\
\frac{1}{l}\left(\mathrm{~A}_{E C} \mathrm{C}+\bar{\Delta}_{E}\right) \\
\frac{1}{l}\left(\mathrm{~A}_{E C} \mathrm{C}+\bar{\Delta}_{E}\right)+\left(-\mathrm{a}_{Z G} G+\bar{\delta}_{Z}\right)
\end{array}\right] }
\end{aligned}
$$


The interface displacements are the traces of the displacements at the substructure's border.

$$
Z_{1}=\mathrm{u}_{1} \quad Z_{2}=\mathrm{u}_{2}
$$

The kinematic unknown is deduced using the finite element derivative operator $B$.

$$
\mathrm{E}=B \mathrm{u}
$$

\section{- The temporal problem leading to $\alpha$}

Given the spatial functions $\left(\mathbf{E}_{p}, \underline{Z}\right)$, one seeks $\alpha(t)$ which minimizes $e_{C R \varepsilon_{\mathrm{p}}, E}^{2}\left(\varepsilon_{\mathrm{p}}, \underline{W}\right)$. The stationarity of the error is expressed by

$$
\forall \alpha^{\star} \int_{I_{i}^{C}} \dot{\alpha}^{\star}\left(a_{11} \dot{\alpha}+a_{10} \alpha-\bar{d}_{1}\right) d t+\int_{I_{i}^{C}} \alpha^{\star}\left(a_{01} \dot{\alpha}+a_{00} \alpha-\bar{d}_{0}\right) d t=0
$$

With the time scheme chosen, this can be written in the form:

$$
\mathbf{T}_{n} \mathbf{q}_{n}=\mathbf{b}_{n}
$$

with $\mathbf{q}_{n}^{T}=\left[\alpha_{n-1} \alpha_{n}\right], \mathbf{b}_{n}^{T}=\left[-\frac{\bar{d}_{1}}{d t}\left(\frac{\bar{d}_{1}}{d t}+\bar{d}_{0}\right)\right]$ and the elementary matrix

$$
\begin{aligned}
& \mathbf{T}_{n}=\left[\begin{array}{cc}
\frac{a_{11}}{d t^{2}} & \left(-\frac{a_{10}}{d t}-\frac{a_{11}}{d t^{2}}\right) \\
\left(-\frac{a_{0}}{d t}-\frac{a_{11}}{d t^{2}}\right) & \left(\frac{a_{11}}{d t^{2}}+\frac{a_{10}}{d t}+\frac{a_{01}}{d t}+a_{00}\right)
\end{array}\right] \\
& a_{11}(t)=\sum_{x=0}^{l}\left(\frac{1}{\mathbf{H}_{\sigma}} \mathrm{E}_{p}^{2}\right) \delta x+\frac{1}{\mathbf{h}^{-}} Z_{1}^{2}+\frac{1}{\mathbf{h}^{-}} Z_{2}^{2} \\
& a_{00}(t)=\sum_{x=0}^{l}\left(\mathbf{H}_{\sigma} \mathrm{C}^{2}\right) \delta x+\mathbf{h}^{-} G_{1}^{2}+\mathbf{h}^{-} G_{2}^{2} \\
& a_{10}(t)=a_{01}(t)=-\sum_{x=0}^{l}\left(\mathrm{E}_{p} \mathrm{C}\right) \delta x+Z_{1} G_{1}+Z_{2} G_{2} \\
& \bar{d}_{1}(t)=\sum_{x=0}^{l} \mathrm{E}_{p} \frac{1}{\mathbf{H}_{\sigma}} \bar{\Delta} \delta x+Z_{1} \frac{1}{\mathbf{h}^{-}} \bar{\delta}+Z_{2} \frac{1}{\mathbf{h}^{-}} \bar{\delta} \\
& \bar{d}_{0}(t)=\sum_{x=0}^{l}\left(\mathrm{C} \bar{\Delta}_{\varepsilon}\right) \delta x+G_{1} \bar{\delta}+G_{2} \bar{\delta}
\end{aligned}
$$

The minimization problems which must be solved in order to use the reduced basis are very similar to the problem of the determination of the new pairs. The only difference is that the spatial functions are known; so no iterative process between time and space is needed. The orthonormalization of the spatial functions leads to simplifications.

\subsubsection{Resolution of the microproblems, cinematic hardening part}

The microproblem 1 consists in finding $\boldsymbol{X}$ and $\boldsymbol{\alpha}$ such that it verify the state law and the search direction

$$
\left\{\begin{array}{l}
\boldsymbol{X}=\frac{2}{3} \mathcal{C} \boldsymbol{\alpha} \\
\dot{\boldsymbol{\alpha}}=\mathbf{H}_{\mathbf{X}} \boldsymbol{X}=\left(k\langle f\rangle_{+}^{\mathcal{N}} \frac{3 \gamma}{2 \mathcal{C}}+k \mathcal{N}\langle f\rangle^{\mathcal{N}-1} C_{1}^{2}\right) \boldsymbol{X}
\end{array}\right.
$$


This simple differential equation in time is solved in each element separately, with the time integration scheme chosen.

\subsubsection{Resolution of the macroproblem}

The macroproblem consists of two steps. First, one must determine operators $\mathbf{L}_{E}$ and $\mathbf{L}_{E^{\prime}}$ (Figure 7 ) using Algorithm 4 Then, one must find the macro equilibrium (Equation [21]).

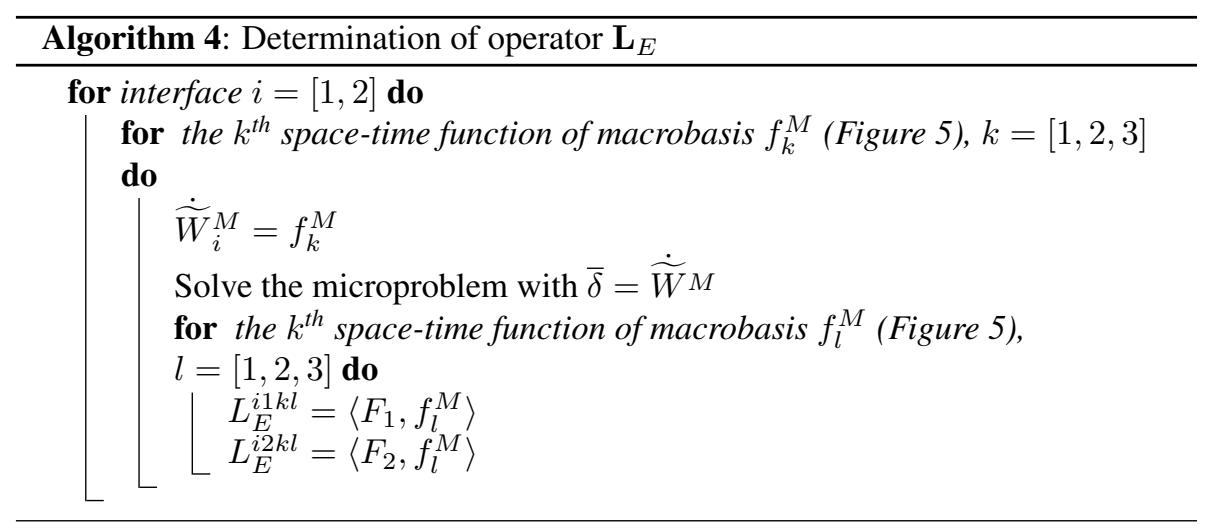

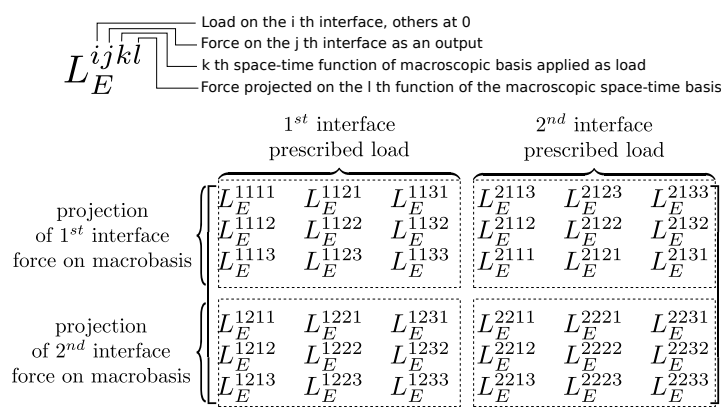

Figure 7. Determination of the homogenized operator for a substructure

In our case, the macro equilibrium can be expressed as

$$
\left[\begin{array}{ccc}
L_{E}^{11} & L_{E}^{21} & 0 \\
L_{E}^{12} & L_{E}^{22}+L_{E^{\prime}}^{11} & L_{E^{\prime}}^{21} \\
0 & L_{E^{\prime}}^{12} & L_{E^{\prime}}^{22}
\end{array}\right]\left[\begin{array}{c}
\dot{\widetilde{W}}^{M} \\
\dot{\widetilde{W}}_{2}^{M} \\
\dot{\widetilde{W}}_{3}^{M}
\end{array}\right]=\left[\begin{array}{c}
F_{1}^{M} \\
F_{2}^{M} \\
F_{3}^{M}
\end{array}\right]
$$

where $L_{E}$ (respectively $L_{E^{\prime}}$ ) is an $n \times n$ (respectively $n^{\prime} \times n^{\prime}$ ) matrix, $n$ (respectively $n^{\prime}$ ) being the sum of the sizes of the macroscopic bases of all the interfaces of substructure $\Omega_{E}$ (respectively $\Omega_{E^{\prime}}$ ); $\dot{\widetilde{W}}_{i}^{M}$ and $F_{i}^{M}$ are vectors of dimension $m$ (the size 
of interface's macrobasis). For example, the first component of $F_{2}^{M}$ is the projection of the macro basis of $-\left(F_{2}+F_{3}\right)$ onto the first temporal function.

Operators $\mathbf{L}_{E}$ and $\mathbf{L}_{E^{\prime}}$ are updated each time the search direction changes. In the one-dimensional example, $2 \times n$ (respectively $2 \times n^{\prime}$ ) microproblems must be solved for substructure $\Omega_{E}$ (respectively $\Omega_{E^{\prime}}$ ) in order to build the macro operator.

\subsection{Summary of the parameters of the method}

The LATIN method introduces fundamental parameters that are the search directions.

The search directions associated with the interface problems, $\mathbf{h}^{+}$and $\mathbf{h}^{-}$are linked to the global rigidity of the substructures and the time of study. We choose to keep the elastic rigidity (Equation [18] in the general frame, and Equation [42] for the 1D example). Search directions at the local and the linear stage are taken equal $\mathbf{h}^{+}=\mathbf{h}^{-}$. In the substructures, for the local search direction operator $\mathbf{H}+$, we choose an operator that preserves dual quantities, i.e. $\hat{\mathbf{f}}=\mathbf{f}$ (Equation [18] in the general frame, and Section 4.5 for the 1D example). For the linear search direction operator $\mathbf{H}^{-}$, we choose an operator associated to the "pseudo-tangent" to $\Gamma$ space (Equation [19] in the general frame, and Section 4.6.1 for the 1D example). This operator is regularized introducing a parameter $\xi$ set in our example to 0.15 (Section 4.6.1). These search directions are similar to the ones used in a Newton tangent algorithm.

To ensure the convergence of the method, a relaxation parameter $\mu$ is introduced, which value is set to 0.8 (see Section 3.8).

The PGD approximation in the linear stage also introduces parameters. Firstly, the choice of the norm to define the constitutive error, for which we take an operator linked to the linear search direction (see Equation [24]. Then a parameter $\xi^{0}$ is introduced to control the enrichment of the approximation. A value of 0.3 is chosen (see Section 3.7.2.

\subsection{Illustrations}

\subsubsection{Convergence of the method}

A reference solution was calculated using a Newton algorithm converged up to the precision of the machine. An error for that solution was calculated in order to verify both the convergence of the multiscale LATIN algorithm and the relevance of the convergence indicator defined in Section 3.8. In Figure 8, one can see that the algorithm converges toward the solution and that the convergence rate can be checked throughout the iterations using the convergence indicator. For a convergence indicator equal to $0.01 \%$ (Figure 9), no visible difference exists compared to the reference solution. The strain is admissible throughout the structure and no displacement gap can be observed at the interfaces. 


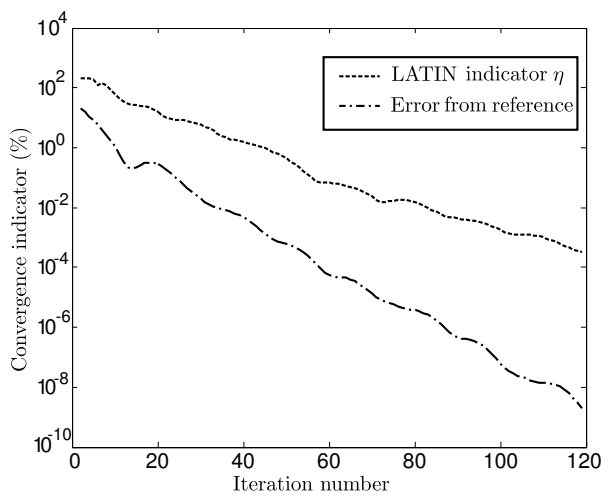

Figure 8. Convergence indicators during the iterations of the algorithm
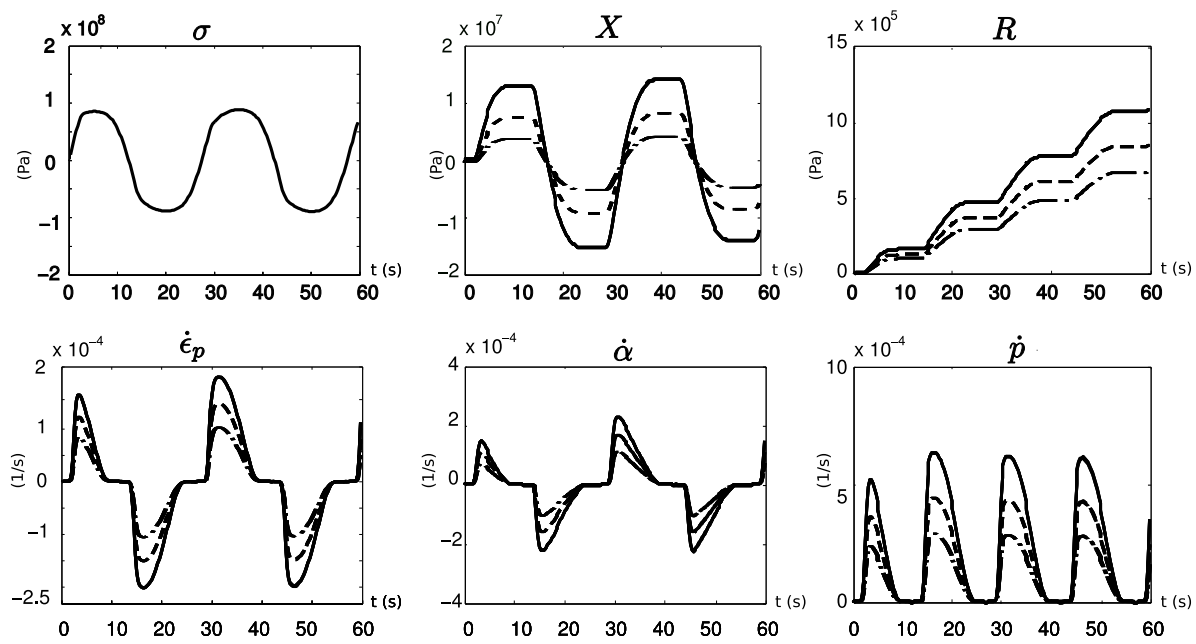

Figure 9. Solution for a convergence indicator $\eta=0.01 \%$ in the 3 materials (-Material 1, - - Material 2, -- Material 3)

\subsubsection{Representation of the fields}

The advantage of the PGD approximation is that only very few pairs are needed in order to represent a field. For example, at Iteration 4 of the algorithm, only one pair was generated in the first bar to represent $\dot{\varepsilon}_{\mathrm{p}}$ (Figure 10 (a)), and the approximation error indicator $\xi$ was $0.5 \%$. At Iteration 30, four pairs were used for an error equal to $0.48 \%$ (Figure 10 (b)), while the convergence indicator $\eta$ of the algorithm was equal 
to $8 \%$. In our example, a maximum of five pairs was generated in each substructure for a convergence indicator equal to $0.0001 \%$.

The generation of a pair requires the resolution of two finite element problems. Altogether, during the whole iteration process of the LATIN method, only ten such finite element problems were solved for each substructure. Conversely, using an incremental technique, at least one finite element problem would have had to be solved for each substructure and at each time step, which, in our case, represents a minimum of sixty finite element problems. Actually, since the problem is nonlinear, the iterations of the Newton algorithm would have represented an even larger number.

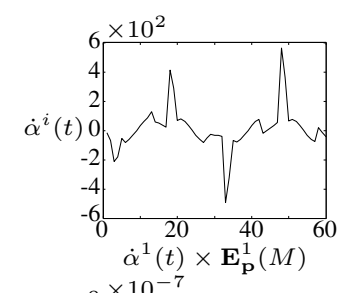

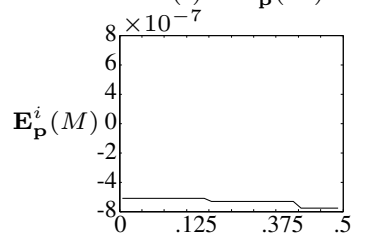

$$
\dot{\varepsilon}_{\mathrm{p}}(M, t)
$$

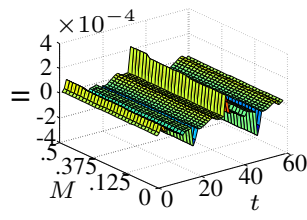

(a)
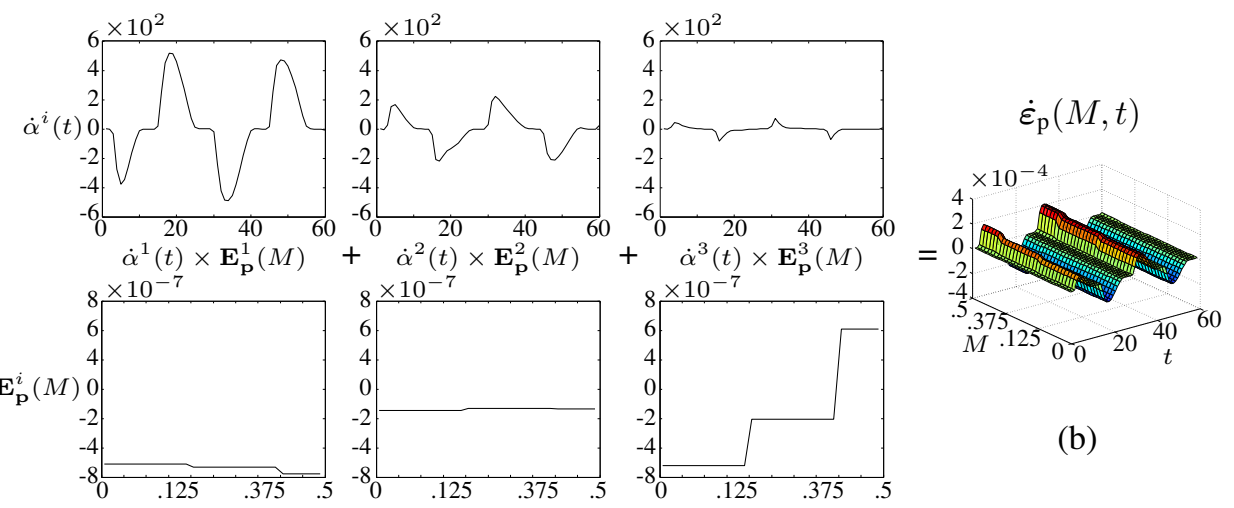

(b)

Figure 10. Number of space-time pairs required to represent $\dot{\varepsilon}_{p}$ in the first bar at Iterations 4 (a) and $30(b)$ of the LATIN method

In 3D problems, the PGD technique enables a drastic reduction in computation time ((Ladevèze et al., 2010) for a viscoelastic constitutive law). In addition, if multiple similar problems need to be solved, one can keep the same basis as long as $\mathbf{A}_{\mathbf{d}}^{*}$ does not change. Then, the iteration of the algorithm in the linear step concerns only the updating of the temporal functions. 


\section{Example of a plate with a hole}

More complex examples can be treated with this method, let consider a plate with a hole. The symmetry conditions allow us to consider an eighth of the plate. The plate is decomposed into four substructures with a total of 1,407 DDLs. A prescribed displacement is imposed on the face opposed to the hole, in the normal to the face direction. The evolution in time of the displacement is a simple ramp, split into 30 time steps (Figure 11. A maximum strain of $0.47 \%$ is obtained with these boundary conditions, with $0.37 \%$ of plastic strain.
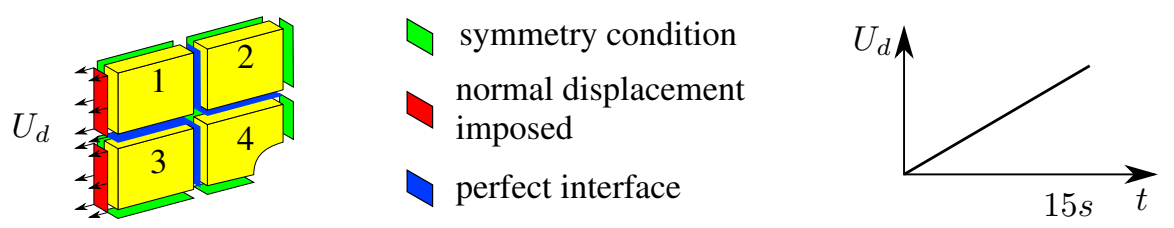

Figure 11. Plate with a hole boundary conditions and domain decomposition

A LATIN error indicator of $10^{-2}$ is reached in 79 iterations (Figure 12). Different numbers of pairs are necessary in the domains to reach this error. The number of pairs is presented in the following table.

\begin{tabular}{|c|c|c|c|c|}
\hline Domain & 1 & 2 & 3 & 4 \\
\hline Number of pairs & 11 & 13 & 19 & 28 \\
\hline
\end{tabular}

Most of the plasticity occurs in domain 4, that why more pairs are necessary in that domain. Domain 1 remains elastic and the combination of the pairs always gives null plastic strain but non zero stress, strain, interface force and displacement.

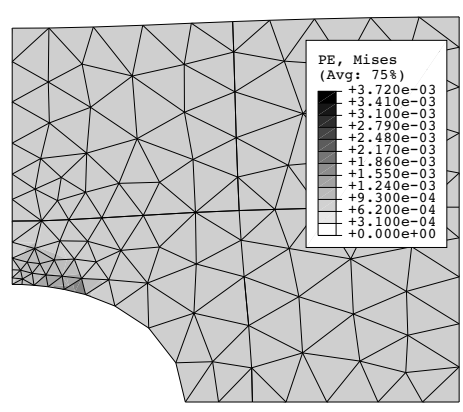

LATIN ITERATION 4

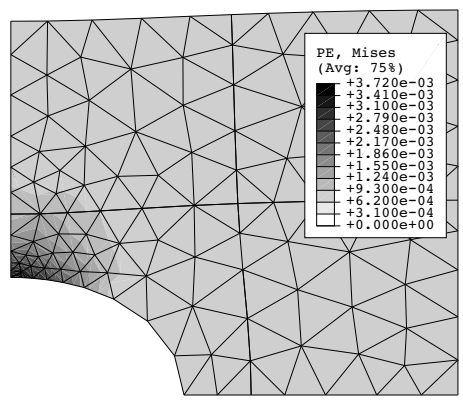

LATIN ITERATION 79

Figure 12. Plastic strain at the fourth and last iterations of the LATIN, last time step. The displacement is magnified by a factor 100 . 


\section{Conclusion}

We presented the details of the application of the multiscale LATIN method to elastic-viscoplastic analysis. In order to use this approach with such a constitutive model, it was necessary to address two main points. The first point is the use of a normal formulation of the constitutive law. We particularized the Marquis-Chaboche elastic-viscoplastic model to this type of formulation. The second point is the careful selection of the method's linear search direction because this operator must be sufficiently regular. We proposed a regularization of the tangent linear search direction using the state law operator.

A fundamental feature of the LATIN method is the use of Proper Generalized Decomposition. Thanks to this technique, only very few finite element problems need to be solved compared to usual incremental techniques. Furthermore, the fact that thanks to the domain decomposition method the strategy is suitable for parallel computing makes the calculation of complex, real-life nonlinear structures truly affordable.

The next step will be the application of this strategy to other problems taking into account variations of the material properties. The multiscale LATIN method enables one to reuse the solution of a problem in order to solve similar problems. That strategy has already been applied very efficiently to parametric studies with multiple contacts (Boucard et al., 2003) and now needs to be extended to the parametric analysis of problems with elastic-viscoplastic behavior.

\section{Acknowledgements}

This work was supported by the Agence Nationale pour la Recherche as part of project MATETPRO07: Approche mécano Probabiliste Pour la conception RObuste en FatIgue (APPROFI).

\section{References}

Ammar A., Mokdad B., Chinesta F., Keunings R., “ A new family of solvers for some classes of multidimensional partial differential equations encountered in kinetic theory modeling of complex fluids", Journal of Non-Newtonian Fluid Mechanics, vol. 139, n³ 3, p. 153-176, 2006.

Aubard X., Boucard P. A., Ladevèze P., Michel S., “ Modeling and simulation of damage in elastomer structures at high strains", Computers \& Structures, vol. 80, n 27-30, p. 22892298, 2002.

Boisse P., Bussy P., Ladeveze P., “ A new approach in non-linear mechanics: The large time increment method", International journal for numerical methods in engineering, vol. 29, $\mathrm{n}^{\circ}$ 3, p. 647-663, 1990.

Boisse P., Ladeveze P., Poss M., Rougee P., " A new large time increment algorithm for anisotropic plasticity", International journal of plasticity, vol. 7, n 1-2, p. 65-77, 1991. 
Boucard P.-A., Champaney L., “ A suitable computational strategy for the parametric analysis of problems with multiple contact", International Journal for Numerical Methods in Engineering, vol. 57, p. 1259-1282, 2003.

Boucard P. A., Ladevèze P., Poss M., Rougée P., “ A nonincremental approach for large displacement problems”, Computers \& Structures, vol. 64, n 1-4, p. 499-508, 1997.

Chinesta F., Ammar A., Lemarchand F., Beauchene P., Boust F., “ Alleviating mesh constraints: Model reduction, parallel time integration and high resolution homogenization", Computer Methods in Applied Mechanics and Engineering, vol. 197, n 5, p. 400-413, 2008.

Cognard J.-Y., Ladevèze P., “ A large time increment approach for cyclic viscoplasticity”, International Journal of Plasticity, vol. 9, n 2, p. 141-157, 1993.

Cognard J. Y., Ladevèze P., Talbot P., “ A large time increment approach for thermo-mechanical problems", Advances in Engineering Software, vol. 30, n 9-11, p. 583-593, 1999.

Devries F., Dumontet F., Duvaut G., Léné F., “ Homogenization and damage for composite structures", International Journal for Numerical Methods in Engineering, vol. 27, p. 285298, 1989.

Farhat C., Chen P. S., Mandel J., “ A Scalable Lagrange Multiplier Based Domain Decomposition Method For Time-Dependent Problems”, International Journal for Numerical Methods in Engineering, vol. 38, n² 22, p. 3831-3853, 1995.

Farhat C., Lesoinne M., Pierson K., “ A scalable dual-primal domain decomposition method”, Numerical Linear Algebra With Applications, vol. 7, n 7-8, p. 687-714, 2000a.

Farhat C., Pierson K., Lesoine M., “ The second generation FETI Methods and their application to the parallel solution of large-scale linear and geometracally non-linear structural analysis problems", Computer Methods in Applied Mechanics and Engineering, vol. 184, p. 333$374,2000 b$.

Farhat C., Roux F.-X., “ A method of Finite Element Tearing and Interconnecting and its parallel solution algorithm", International Journal for Numerical Methods in Engineering, vol. 32, p. 1205-1227, 1991.

Feyel F., " A multilevel finite element (FE2) to describe the response of highly non-linear structures using generalized continua”, Computer Methods in Applied Mechanics and Engineering, vol. 192, p. 3233-3244, 2003.

Fish J., Shek K., Pandheeradi M., Shephard M. S., " Computational plasticity for composite structures based on mathematical homogenization: Theory and practice", Computer Methods in Applied Mechanics and Engineering, vol. 148, p. 53-73, 1997.

Gosselet P., Rey C., " Non-overlapping domain decomposition methods in structural mechanics”, Archives of Computational Methods in Engineering, vol. 13, p. 515-572, 2006.

Gosselet P., Rey C., Léné F., Dasset P., " A domain decomposition method for quasiincompressible formulation with discontinuous pressure fields", Revue Européenne des Eléments Finis, vol. 11, p. 363-378, 2002.

Ladevèze P., " New algorithms: mechanical framework and development (in french)", Compte rendu de l'académie des Sciences, vol. 300(2), p. 41-44, 1985.

Ladevèze P., "New advances in the large time increment method", New advances in computational structural mechanics, P. Ladevèze and O.C. Zienkiewicz, 1992.

Ladevèze P., Nonlinear Computationnal Structural Mechanics - New Approaches and NonIncremental Methods of Calculation, Springer Verlag, 1999. 
Ladeveze P., Cognard J., Talbot P., A Non-incremental and Adaptive Computational Approach in Thermo-viscoplasticity, p. 281-291, 2002a.

Ladevèze P., Loiseau O., Dureisseix D., “ A micro-macro and parallel computational strategy for highly heterogeneous structures”, International Journal for Numerical Methods in Engineering, vol. 52, p. 121-138, 2001.

Ladevèze P., Néron D., Gosselet P., “ On a mixed and multiscale domain decomposition method", Computer Methods in Applied Mechanics and Engineering, vol. 196, n ${ }^{\circ} 8$, p. 1526-1540, 2007.

Ladevèze P., Nouy A., Loiseau O., “ A multiscale computational approach for contact problems", Computer Methods in Applied Mechanics and Engineering, vol. 191, p. 4869-4891, 2002b.

Ladevèze P., Passieux J.-C., Néron D., “ The LATIN multiscale computational method and the Proper Generalized Decomposition”, Computer Methods in Applied Mechanics and Engineering, vol. 199, p. 1287-1296, 2010.

Le Tallec P., De Roeck Y.-H., Vidrascu M., “ Domain-decomposition methods for large linearly elliptic three dimensional problems", Journal of Computational and Applied Mathematics, vol. 34, p. 93-117, 1991.

Lemaitre J., Chaboche J., Mechanics of solid materials, Cambridge Univ Pr, 1994.

Magoulès F., Roux F.-X., “ Lagrangian formulation of domain decomposition methods: A unified theory”, Applied Mathematical Modeling, vol. 30, p. 593-615, 2006.

Mandel J., " Balancing domain decomposition", Communications in Numerical Methods in Engineering, vol. 9, n 233-241, p. 233-241, 1993.

Néron D., Dureisseix D., “ A computational strategy for thermo-poroelastic structures with a time-space interface coupling", International Journal for Numerical Methods in Engineering, vol. 75, n 9, p. 1053-1084, 2008.

Néron D., Ladevèze P., “ Proper Generalized Decomposition for multiscale and multiphysics problems", Archives of Computational Methods in Engineering, vol. 17, p. 351-372, 2010.

Nouy A., " A generalized spectral decomposition technique to solve a class of linear stochastic partial differential equations", Computer Methods in Applied Mechanics and Engineering, vol. 196, p. 4521-4537, 2007.

Nouy A., Ladevèze P., " On a multiscale computational strategy with time and space homogenization for structural mechanics", Computer Methods in Applied Mechanics and Engineering, vol. 192, p. 3061-3087, 2004.

Oden J. T., Vemaganti K., Moës N., " Hierarchical modeling of heterogeneous solids", Computer Methods in Applied Mechanics and Engineering, vol. 172, p. 3-25, 1999.

Passieux J.-C., Ladevèze P., Néron D., “ A scalable time-space multiscale method: adaptive time scales separation”, Computational Mechanics, vol. 199, p. 1287-1296, 2010.

Risler F., Rey C., “ Iterative accelerating algorithms with Krylov subspaces for the solution to large-scale nonlinear problems", Numerical Algorithms, vol. 23, p. 1-30, 2000.

Sanchez-Palencia E., "Comportement local et macroscopique d'un type de milieux physiques hétérogènes”, International Journal of Engineering Science, vol. 12, n 4, p. 331-351, 1974.

Wriggers P., Nonlinear Finite Element Methods, Springer Verlag, 2008.

Zohdi T., Oden J., Rodin G., “ Hierarchical modeling of heterogeneous bodies”, Computer Methods in Applied Mechanics and Engineering, vol. 138, n 1-4, p. 273-298, 1996.

Received: 18 March 2011

Accepted: 19 October 2011 
